\title{
Novel and Efficient Palladium Complexes with $\beta$-Ketoiminate Ligands for the Polymerization of Norbomene
}

\author{
Dong Hwan Lee, Yoon Joo Hwang, Jin San Yoon, ${ }^{\dagger}$ Moon Kun Choi, ${ }^{\ddagger}$ and Ik-Mo Lee \\ Department of Chemistry, Inha Lniversity, Incheon $402-751$, Korea. ${ }^{*}$ E-mail: imlee ainha ac kr \\ Department of Polwmer Engineering. Inha Chiversin, Incheon $402-751$, Korea \\ ${ }^{\ddagger}$ Department of Chemistry, Ionsei Lniversity, Seoul 120-749, Korea \\ Received December 5, 2008. Accepted January 17, 2009
}

\begin{abstract}
A series of the noble palladium complexes containing $\beta$-ketoiminate ligands with internal bases, $\left[\mathrm{Pd}\left(\eta^{3}{ }^{3}\right.\right.$-allyl $)(\beta-$ ketoiminate $)],\left[\mathrm{Pd}(\mathrm{Me})\left(\mathrm{PPh}_{3}\right)(\beta\right.$-ketoiminate $\left.)\right]$ and $[\mathrm{Pd}(\mathrm{Me})(\beta$-ketoiminate $)]$, have been successfully prepared. Crystallographically determined structures showed that these complexes are distorted square planar and pendant bases of the $\beta$-ketoiminate ligands fail to coordinate to the metal in the first two classes of complexes while bases do coordinate in the $3^{\text {rd }}$ class complexes. These complexes are active towards norbornene polymerization on activation with $\mathrm{H}\left(\mathrm{OEt}{ }_{2}\right)_{2} \mathrm{BAr}_{4}\left(\mathrm{Ar}^{\prime}=3,5\right.$-bistrifluoromethylphenyl) and modified methylalumioxane (MMAO). MMAO is more efficient for the activation for polymerization. Generally, the polymerization activity increases with the following order; $[\mathrm{Pd}($ allyl $)(\beta$-ketoininate $)]<\left[\mathrm{Pd}(\mathrm{Me})\left(\mathrm{PPh}_{3}\right)(\beta\right.$-ketoiminate $\left.)\right]<[\mathrm{Pd}(\mathrm{Me})(\beta$-ketoiminate $)]$.
\end{abstract}

Key Words: Palladium complexes. $\beta$-Ketoiminate ligands. Norbornene polymerization. Activators. Structures

\section{Introduction}

Up to date. polyolefins have been mainly produced with early transition metal-based cataly sts. Recently' late transition metals. in particular nickel and palladium, have attracted $\mathrm{m}$ uch attention as olefin polymerization catalysts due to their less electrophilicity and greater heteroatom tolerance ${ }^{1.2}$ even though some group 10 transition metal complexes. especially $\left[\mathrm{P}_{2} \mathrm{M}\right]^{\mathrm{j}^{+}}[\mathrm{A}]_{-}^{-}$(P: diphosphine: $\mathrm{M}=$ Pd. Pt: $\mathrm{A}^{-}=\mathrm{OTf}^{-}, \mathrm{BF}_{+}^{-}$. $\mathrm{PF}_{6}^{-}$. $\mathrm{SbF}_{6}^{-} . \mathrm{ClO}_{4}^{-}$). act as excellent Lewis acid catalysts. ${ }^{3}$ Early pioneering works by Keim $e t a I^{4}$ initiated the research in this field and discrete $\mathrm{Ni}$ and Pd monoalkyl complexes containing $\alpha$-diimines prepared by Brookhart highlighted key features in the olefin polymerization catalyzed by late transition metal complexes. "Grubbs" neutral $\mathrm{Ni}$ complexes with salicylaldiminato ligands were followed to produce lighly linear polyethylene at a rate comparable with highly active metallocene catalysts. ${ }^{6}$ Many catalytic systems have been followed and they generally contain bidentate $\mathrm{P}^{\wedge} \mathrm{O}, \mathrm{N}^{\wedge} \mathrm{O}, \mathrm{P}^{\wedge} \mathrm{P}$ and $\mathrm{N}^{\wedge} \mathrm{N}$ ligands with bulky aryl substituents. ${ }^{1,3}$ However. late transition metal complexes with tri or higher dentate ligands are generally known to be inactive or less active for olefin poly merizations than ones with bidentate ligands."

On the other hand, norbomene and its derivatives have drawn much interest due to their polymers showing unique physical properties such as high glass transition temperature. optical transparency. low dielectric properties and birefringence. which can be applicable in the new optical information storage media, microelectronics, packing and gas separation. ${ }^{9.11}$ Norbomene is known to be polymerized by ringopening metathesis polymerization (ROMP). cationic polymerization and vinyl addition polymerization. Since the first adoption of a $\mathrm{TiCl}_{4} / \mathrm{Al}^{\prime} \mathrm{Bu}_{3}$ catalyst. various catalysts from $\mathrm{Ni}$. $\mathrm{Co} . \mathrm{Cr}, \mathrm{Ti} . \mathrm{Zr}$ and $\mathrm{Pd}$ complexes have been used for norbornene vinyll polymerization and recently well reviewed. ${ }^{11}$ Some of these complexes are active towards olefin polymeri- zation without cocatalysts ${ }^{\text {ka.1.12 }}$ but most of them require cocataly'sts such as phosphine scavengers. methylaluminoxane (MAO), boranes with or without alkylaluninum, and borates. ${ }^{12}$

Recently. some early and late transition metal complexes with $\beta$-ketoiminate or $\beta$-diketininate, a bidentate monoanionic ligand have been prepared and tested for the olefin polymerization. ${ }^{\gamma(a), 1+}$ Among these complexes, bis( $\beta$-ketoiminato) $\mathrm{Ni}(\mathrm{II})$ complexes $\mathrm{s}^{13 ! e 1}$ are active for the polymerization of even polar monomers such as MMA. Since we have been involved in the research for the synthesis of group 4 and 5 complexes containing $\mathrm{N}$-alkoxy- $\beta$-ketoiminate. a terdentate dianionic ligand as pronising metalloorganic chemical vapor deposition (MOCVD) precursors. ${ }^{15}$ we have kept an eye on $\mathrm{Ni}$ and $P d$ complexes which are formulated as $\left(\mathrm{N}^{\wedge} \mathrm{O}\right) \mathrm{MR}(\mathrm{L})(\mathrm{M}=$ $\mathrm{Ni}, \mathrm{Pd} ; \mathrm{L}=$ neutral ligands) and showed high activities towards olefin polymerization. The outcome would be interesting if $\mathrm{L}$ is connected to the $\mathrm{N}^{\wedge} \mathrm{O}$ system because $\mathrm{L}$ should be replaced with incoming olefin monomer during the process of polymerization. Since $\beta$-ketoiminate complexes have drawn interest due to their higher thermal stability than the $\beta$-diketonate analogues and their versatility. by changing the imine substituents, for tailoring their reactivity and volatility. ${ }^{16}$ some palladium complexes containing $\dot{\beta}$-ketoiminate derivatives. one of $\mathrm{N}^{\wedge} \mathrm{O}$ type ligands with pendant bases have been prepared to test their potential application as olefin poly merization cataly sts.

Herein we report the novel and well-characterized palladium complexes containing tridentate monoanionic functional $\beta$-ketoiminate ligands. which are highly active towards norbornene and ethylene.

\section{Expenimental Section}

All the works involving moisture-sensitive conpounds were carried out using standard Schlenk or dry-box techniques. All reagents, purchased from Aldrich Chemical Co., 
were used as supplied commercially without further purification. ${ }^{1} \mathrm{H}$ and ${ }^{17} \mathrm{C}\left\{{ }^{1} \mathrm{H}\right\}$ NMR spectra were recorded by using $5 \mathrm{~mm}$ tube on a Varian Unity Inova $400(400.256$ and $100.657 \mathrm{MHz}$. respectively) or a Varian Gemini 2000 (199.976 and $50.289 \mathrm{MHz}$, respectively) spectrometer and were referenced to tetramethylsilane (TMS). ${ }^{3 \mathrm{l}} \mathrm{P}\left\{{ }^{\mathrm{j}} \mathrm{H}\right\} \mathrm{NMR}$ spectra were recorded on a Varian ( $162.027 \mathrm{MHz}$ ) FT-NMR spectrometer and were referenced to external $\mathrm{PPh}_{3}(-5.3$ ppm relative to $85 \% \mathrm{H}_{3} \mathrm{PO}_{4}$ ). All manipulations were conducted under an inert atmosphere. Elemental analyses were performed with EA-1110 (CE Instruments) in the Inha University.

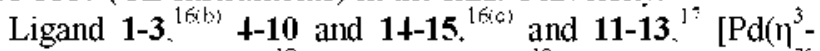
$\left.\left.\mathrm{CH}_{2} \mathrm{CMeCH}_{2}\right)(\mu-\mathrm{Cl})\right]_{2},{ }^{18}\left[\mathrm{Pd}\left(\mathrm{PPh}_{3}\right) \mathrm{MeCl}\right]_{2}^{19}[\mathrm{Pd}(\mathrm{COD}) \mathrm{MeCl}]^{301}$ $\mathrm{H}\left(\mathrm{OEt}_{2}\right)_{2} \mathrm{BAr}_{4}{ }_{4}$ and NaBAr'${ }_{4}\left(\mathrm{Ar}^{\prime}=3.5-\left(\mathrm{CF}_{3}\right)_{2} \mathrm{C}_{6} \mathrm{H}_{3}\right)^{21}$ have been prepared according to literature procedures. Complexes $\mathbf{1 b}$ and $4 \mathrm{~b}$ were already reported elsewhere. MMAO (Tosoh Finechem Co.. 5.7\% Al content in toluene) was used as supplied.

Synthesis of complexes.

$\mathrm{Pd}\left(\eta^{3}-\mathrm{CH}_{2} \mathrm{CMeCH}_{2}\right)\left(\mathrm{CH}_{3} \mathrm{C}(\mathrm{O}) \mathrm{CHC}\left(\mathrm{NCH}_{2} \mathrm{CH}_{2} \mathrm{CH}_{2}\right.\right.$ $\left.\left.\mathrm{OCH}_{3}\right) \mathrm{CH}_{3}\right)(\mathbf{1 a})$ : A mixture of distilled diethyl ether $(30 \mathrm{~mL})$. $\mathrm{Tl}\left(\mathrm{OC}_{2} \mathrm{H}_{5}\right)(0.63 \mathrm{~g} .2 .5+\mathrm{mmol})$ and Ligand $\mathrm{CH}_{3} \mathrm{C}(\mathrm{O}) \mathrm{CHC}$ $\left(\mathrm{NHCH}_{2} \mathrm{CH}_{2} \mathrm{CH}_{2} \mathrm{OCH}_{3}\right) \mathrm{CH}_{3}(0.4+\mathrm{g} .2 .54 \mathrm{mmol})$ was stirred at room temperature for $\mathrm{lhr}$ and then the solvent was removed in vacuum. After the above mixture added to diethyl ether and was stirred at $-20^{\circ} \mathrm{C}$ and then added to suspension of $\left[\left\{\mathrm{Pd}\left(\eta^{3}-\right.\right.\right.$ $\left.\left.\left.\mathrm{CH}_{2} \mathrm{CMeCH}_{2}\right)(\mu-\mathrm{Cl})\right\}_{2}\right](0.50 \mathrm{~g} 1.27 \mathrm{mmol})$ in diethyl ether $(40 \mathrm{~mL})$. After it was stirred at $-20^{\circ} \mathrm{C}$ for $2 \mathrm{~h}$, the mixture was filtered through celite on a frit into a Schlenk flask at $-20^{\circ} \mathrm{C}$ and then the solvent was removed in Facum and washed with distilled hexane and dried in vacuum to give a pale yellow solid. (yield: $0.53 \mathrm{~g} .63 \%$ )

${ }^{l} \mathrm{H}-\mathrm{NMR}\left(199.976 \mathrm{MHz}, \mathrm{CDCl}_{3}\right): \hat{o} 4.77(\mathrm{~s}, \mathrm{lH}, \mathrm{C}(\mathrm{O}) \mathrm{CH}=\mathrm{C}(\mathrm{N})$ ), 3.77 (t. 2H. $\left.\mathrm{J}_{\mathrm{HH}}=7.0 \mathrm{~Hz}, \mathrm{NCH}_{2} \mathrm{CH}_{2} \mathrm{CH}_{2} \mathrm{OCH}_{3}\right) .3 .59$ (d. $1 \mathrm{H}$. $\mathrm{J}_{\mathrm{HH}}=2.6 \mathrm{~Hz}$, allyl). $3.37\left(\mathrm{t}, 2 \mathrm{H}, \mathrm{J}_{\mathrm{HH}}=5.8 \mathrm{~Hz}\right.$. $\mathrm{NCH}_{2} \mathrm{CH}_{2} \mathrm{CH}_{2}-$ $\mathrm{OCH}_{3}$ ). 3.32(s. $3 \mathrm{H} . \mathrm{NCH}_{2} \mathrm{CH}_{2} \mathrm{CH}_{2} \mathrm{OCH}_{3}$ ). $3.00\left(\right.$ d. $\mathrm{IH} . \mathrm{J}_{\mathrm{HH}}=$ $2.6 \mathrm{~Hz}$. allyl $), 2.87(\mathrm{~s}, 1 \mathrm{H}$. allyl $), 2.66(5,1 \mathrm{H}$, allyl $), 2.15(5,3 \mathrm{H}$, $\mathrm{CH}_{3}$ of allyl). 1.97 (s. $\left.3 \mathrm{H} . \mathrm{C}(\mathrm{N}) \mathrm{CH}_{3}\right), 1.93$ (s. $3 \mathrm{H}, \mathrm{CH}_{3} \mathrm{C}(\mathrm{O})$ ). $1.87\left(\mathrm{~m} .2 \mathrm{H}, \mathrm{NCH}_{2} \mathrm{CH}_{2} \mathrm{CH}_{2} \mathrm{OCH}_{3}\right) ;{ }^{13} \mathrm{C}-\mathrm{NMR}(50.289 \mathrm{MHz}$. $\left.\mathrm{CDCl}_{3}\right): \delta 176.60\left(\mathrm{~s} . \mathrm{CH}_{3} \mathrm{C}(\mathrm{O}) \mathrm{CH}\right) .164 .55\left(\mathrm{~s}, \mathrm{CH}=\mathrm{C}(\mathrm{N}) \mathrm{CH}_{3}\right)$. $130.54\left(\mathrm{~s} . \mathrm{CH}_{2} \mathrm{C}\left(\mathrm{CH}_{3}\right) \mathrm{CH}_{2}\right), 97.76(\mathrm{~s} . \mathrm{C}(\mathrm{O}) \mathrm{CH}=\mathrm{C}(\mathrm{N})) .70 .33$ (s, $\left.\mathrm{NCH}_{2} \mathrm{CH}_{2} \mathrm{CH}_{2} \mathrm{OCH}_{3}\right) .59 .02\left(5, \mathrm{CH}_{2}\right.$ of allyl), $58.78(\mathrm{~s}$. $\mathrm{NCH}_{2} \mathrm{CH}_{2} \mathrm{CH}_{2} \mathrm{OCH}_{3}$ ), 57.32(s. $\left.\mathrm{NCH}_{2} \mathrm{CH}_{2} \mathrm{CH}_{2} \mathrm{OCH}_{3}\right), 53.77$ (5, $\mathrm{CH}_{2}$ of allyl). 32.16(s. $\left.\mathrm{NCH}_{2} \mathrm{CH}_{2} \mathrm{CH}_{2} \mathrm{OCH}_{3}\right) .26 .82\left(\mathrm{~s} . \mathrm{CH}_{3}\right.$ $\mathrm{C}(\mathrm{O}) \mathrm{CH}$ ) $23.6 \mathrm{l}$ (s. $\mathrm{CH}_{3}$ of allỵl). 21.07(s. $\left.\mathrm{CH}=\mathrm{C}(\mathrm{N}) \mathrm{CH}_{3}\right)$ : Anal. Calcd. For $\mathrm{C}_{13} \mathrm{H}_{23} \mathrm{NO}_{2} \mathrm{Pd}: \mathrm{C} .47 .07: \mathrm{H}, 6.99: \mathrm{N}, 4.22$. Found: C. $47.24 ; \mathrm{H}, 7.30: \mathrm{N}, 4.01$.

Complexes 4a-12a $4 \mathbf{b - 1 3 b}$ and 12c-15c were prepared according to the procedures described for complex $1 \mathbf{a}, \mathbf{1 b}$ and $6 c$, respectively

$\mathrm{Pd}\left(\boldsymbol{\eta}^{3}-\mathrm{CH}_{2} \mathrm{CMeCH}_{2}\right)\left(\mathrm{CH}_{3} \mathrm{C}(\mathrm{O}) \mathrm{CHC}\left(\mathrm{NCH}_{2} \mathrm{C}_{6} \mathrm{H}_{4} \mathrm{OCH}_{3}\right)-\right.$ $\mathbf{C H}_{3}$ ) (4a): yield: $0.63 \mathrm{~g} .65 \%$. ${ }^{\mathrm{l}} \mathrm{H}-\mathrm{NMR}(199.976 \mathrm{MHz}$. $\mathrm{CDCl}_{3}$ ): ò $7.23 \sim 7.18\left(\mathrm{~m}, 2 \mathrm{H}, \mathrm{NCH}_{2} \mathrm{C}_{6} \mathrm{H}_{4} \mathrm{OCH}_{3}\right) .6 .9 \mathrm{l}(\mathrm{t} . \mathrm{IH}$. $\left.\mathrm{J}_{\mathrm{HH}}=3.8 \mathrm{~Hz}, \mathrm{NCH}_{2} \mathrm{C}_{6} H_{4} \mathrm{OCH}_{3}\right), 6.85\left(\mathrm{~d} .1 \mathrm{H}, \mathrm{J}_{\mathrm{HH}}=4.0 \mathrm{~Hz}\right.$. $\left.\mathrm{NCH}_{2} \mathrm{C}_{6} H_{4} \mathrm{OCH}_{3}\right), 4.95\left(\mathrm{~d}, 1 \mathrm{H}, \mathrm{J}_{\mathrm{HH}}=6.6 \mathrm{~Hz} . \quad \mathrm{NCH}_{2} \mathrm{C}_{6} \mathrm{H}_{4}-\right.$ $\left.\mathrm{OCH}_{3}\right), 4+1(5,1 \mathrm{H}, \mathrm{C}(\mathrm{O}) \mathrm{CH}=\mathrm{C}(\mathrm{N})), 3.85\left(\mathrm{~s}, 3 \mathrm{H} . \mathrm{NCH}_{2} \mathrm{C}_{6} \mathrm{H}_{4-}-\right.$ $\left.\mathrm{OCH}_{3}\right), 3.50\left(\mathrm{~d}, 1 \mathrm{H}, \mathrm{J}_{\mathrm{HH}}=1.4 \mathrm{~Hz}\right.$, allyl). $2.75(\mathrm{~s} .1 \mathrm{H}$. ally $)$, $2.48\left(\mathrm{~d} .1 \mathrm{H} . \mathrm{J}_{\mathrm{HH}}=1.2 \mathrm{~Hz}\right.$ allỵl). 2.34(s. $\mathrm{HH}$. allyl). 2.03 (s. $3 \mathrm{H}$.
$\mathrm{CH}_{3}$ of allyl), $1.85\left(\mathrm{~s}, 3 \mathrm{H} . \mathrm{C}(\mathrm{N}) \mathrm{C} H_{3}\right), 1.87\left(\mathrm{~s} .3 \mathrm{H}, \mathrm{CH}_{3} \mathrm{C}(\mathrm{O})\right)$; ${ }^{13} \mathrm{C}-\mathrm{NMR}\left(50.289 \mathrm{MHz}, \mathrm{CDCl}_{2}\right):$ ò $177.34\left(\mathrm{~s} . \mathrm{CH}_{3} \mathrm{C}(\mathrm{O}) \mathrm{CH}\right)$, 166.21 (s. $\left.\mathrm{CH}=\mathrm{C}(\mathrm{N}) \mathrm{CH}_{3}\right), 156.21,128.70 .127 .36 .127 .00$. $120.58,109.56\left(\mathrm{~s} . \mathrm{NCH}_{2} \mathrm{C}_{6} \mathrm{H}_{4} \mathrm{OCH}_{31}, 98.22\right.$ (s. $\mathrm{C}(\mathrm{O})(\mathrm{CH}=\mathrm{C}(\mathrm{N}))$. 58.08 (s. $\left.\mathrm{NCH}_{2} \mathrm{C}_{6} \mathrm{H}_{4} \mathrm{OCH} \mathrm{H}_{2}\right) .58 .00$ (s. $\mathrm{CH}_{2}$ of allyl). $55.36(\mathrm{~s}$. $\left(\mathrm{H}_{2}\right.$ of allyl). 54.83(s. NCH $\left.\mathrm{C}_{4} \mathrm{H}_{4} \mathrm{OCH}_{3}\right), 27.08\left(\mathrm{~s}, \mathrm{CH}_{3} \mathrm{C}(\mathrm{O})\right.$ $\mathrm{CH}), 23.22\left(\mathrm{~s} .3 \mathrm{H} . \mathrm{CH}_{3}\right.$ of allyl). $21.35\left(\mathrm{~s}, \mathrm{CH}=\mathrm{C}(\mathrm{N}) \mathrm{CH}_{3}\right)$ : Anal. Calcd. For $\mathrm{C}_{1}: \mathrm{H}_{3} \mathrm{NO}$ Pd: C. 53.76: H. 6.10: N. 3.69. Found: C. 53.93: H, 6.27: N. 3.33 .

$\mathrm{Pd}\left(\eta^{3}-\mathrm{CH}_{2} \mathrm{CMeCH}_{2}\right)\left(\mathrm{CH}_{3} \mathrm{C}(\mathrm{O}) \mathrm{CHC}\left(\left(\mathrm{NCH}_{2} \mathrm{CH}_{2} \mathrm{C}_{5}-\right.\right.\right.$ $\left.\left.\mathrm{H}_{4} \mathrm{~N}\right) \mathrm{CH}_{3}\right)\left(\mathrm{C}_{5} \mathrm{H}_{4} \mathrm{~N}=2\right.$-pyridyl) $(6 \mathrm{a})$ : yield $0.66 \mathrm{~g} .71 \%$. ${ }^{\mathrm{l}} \mathrm{H}-$ NMR (199.976 MHz, CDCl $)$ : ò 8.54(d. IH. J $\mathrm{HH}_{\mathrm{H}}=4.6 \mathrm{~Hz}, \mathrm{CH}$ of Pyridine), 7.57 (dt. $1 \mathrm{H}, \mathrm{J}_{\mathrm{HH}}=1.4,7.6 \mathrm{~Hz}$. $\mathrm{CH}$ of Pyridine), $7.16 \sim 7.08(\mathrm{~m}, 2 \mathrm{H}, \mathrm{CH}$ of Pyridine). $4.76(\mathrm{~s} . \mathrm{HH}, \mathrm{C}(\mathrm{O}) \mathrm{CH}=$ $\mathrm{C}(\mathrm{N})) .4 .06\left(\mathrm{dt}, 2 \mathrm{H}, \mathrm{J}_{\mathrm{HH}}=2.8 .8 .8 \mathrm{~Hz}, \mathrm{NCH}_{2} \mathrm{CH}_{2}\right.$ Pyridine $) .3 .62$ (br s. H of allyl). 3.14(br s, $\mathrm{H}$ of ally l), 3.05 (t. $2 \mathrm{H} . \mathrm{J}_{\mathrm{HH}}=9.0 \mathrm{~Hz}$, $\mathrm{NCH}_{2} \mathrm{CH}_{2} \mathrm{Py}$ ridine). 2.88 (br s. $\mathrm{H}$ of allyl). 2.71 (br s. $\mathrm{H}$ of allyl). $2.17\left(\mathrm{~s}, 3 \mathrm{H} . \mathrm{C} \mathrm{H}_{3}\right.$ of allyl), $1.98\left(\mathrm{~s} .3 \mathrm{H}, \mathrm{C}(\mathrm{N}) \mathrm{CH} \mathrm{H}_{3}\right) .1 .85(\mathrm{~s}$, 3H. $\left.\mathrm{CH}_{3} \mathrm{C}(\mathrm{O})\right):{ }^{15} \mathrm{C}-\mathrm{NMR}\left(50.289 \mathrm{MHz}^{\circ} \mathrm{CDCl} \mathrm{C}_{3}\right):$ oे 176.73 (s. $\left.\mathrm{CH}_{3} \mathrm{C}(\mathrm{O}) \mathrm{CH}\right), 164.63\left(\mathrm{~s}, \mathrm{CH}=\mathrm{C}(\mathrm{N}) \mathrm{CH}_{3}\right) .159 .50 .149 .65$, $136.41,123.57 .121 .53$ (s Pyridine), 130.72 (s. $\mathrm{CH}_{2} \mathrm{C}\left(\mathrm{CH}_{3}\right) \mathrm{CH}_{2}$ ). $98.23(\mathrm{~s} . \mathrm{C}(\mathrm{O}) \mathrm{CH}=\mathrm{C}(\mathrm{N})), 60.26\left(\mathrm{~s} . \mathrm{NCH} \mathrm{CH}_{2} \mathrm{Py}\right.$ ridine $) .59 .10$ (s, $\mathrm{CH}_{2}$ of allyl), 54.23(s, $\mathrm{CH}_{2}$ of allyl), $40.72\left(\mathrm{~s}, \mathrm{NCH}_{2} \mathrm{CH}_{2}-\right.$ Pyridine). 26.99(s. $\mathrm{CH}_{3} \mathrm{C}(\mathrm{O}) \mathrm{CH}$ ). 23.8l(s, $\mathrm{CH}_{2}$ of allỵl). $21.28\left(\mathrm{~s} . \mathrm{CH}=\mathrm{C}(\mathrm{N})\left(\mathrm{H}_{3}\right)\right.$; Anal. Calcd. For $\mathrm{C}_{14} \mathrm{H}_{22} \mathrm{~N}_{2} \mathrm{OPd}$ : C, 52.68: H. 6.08: N. 7.68. Found: C. 52.82: H. 6.15: N. 7.72.

$\mathrm{Pd}\left(\eta^{3}-\mathrm{CH}_{2} \mathrm{CMeCH}_{2}\right)\left(\mathrm{PhC}(\mathrm{O}) \mathrm{CHC}\left(\mathrm{NCH}_{2} \mathrm{CH}_{2} \mathrm{CH}_{2} \mathrm{OCH}_{3}\right)-\right.$ Ph) (7a): yield: 0.71g, 61\%. ${ }^{\mathrm{l}} \mathrm{H}-\mathrm{NMR}\left(199.976 \mathrm{MHz}, \mathrm{CDCl}_{3}\right)$; o $7.78 \sim 7.71,7.42 \sim 7.24\left(\mathrm{~m}, 10 \mathrm{H}, \mathrm{C}_{6} H_{5} \mathrm{C}(\mathrm{O}) \mathrm{CH}(\mathrm{N}) \mathrm{C}_{6} H_{5}\right)$. $5.37(\mathrm{~s}, 1 \mathrm{H}, \mathrm{C}(\mathrm{O}) \mathrm{CH}=\mathrm{C}(\mathrm{N})) .3 .74\left(\mathrm{~d} .1 \mathrm{H} . \mathrm{J}_{\mathrm{HH}}=2.8 \mathrm{~Hz}\right.$. allyl), 3.57 (dt. $2 \mathrm{H}, \mathrm{J}_{\mathrm{HH}}=1.8 .8 .0 \mathrm{~Hz} . \mathrm{NCH}_{2} \mathrm{CH}_{2} \mathrm{CH}_{2} \mathrm{OCH}_{2}$ ). $3.20 \sim$ $3.14\left(\mathrm{~m}, 6 \mathrm{H}, \mathrm{NCH}_{2} \mathrm{CH}_{2} \mathrm{CH}_{2} \mathrm{OCH}_{3}, \mathrm{NCH}_{2} \mathrm{CH}_{2} \mathrm{CH}_{2} \mathrm{OCH}_{3}\right.$. $\mathrm{H}$ of allyl $), 3.05\left(\mathrm{~s}, 1 \mathrm{H}\right.$, allyl). $2.74(\mathrm{~s} . \mathrm{H}$. allyl $), 2.22\left(\mathrm{~s}, 3 \mathrm{H}, \mathrm{CH}_{3}\right.$ of allyl). $1.85\left(\mathrm{~m}, 2 \mathrm{H}, \mathrm{NCH}_{2} \mathrm{CH}_{2} \mathrm{CH}_{2} \mathrm{OCH}_{3}\right):{ }^{13} \mathrm{CNMR}(50.289$ $\left.\mathrm{MHz}, \quad \mathrm{CDCl}_{3}\right) ; \delta 172.54(\mathrm{~s} . \quad \mathrm{Ph}(\mathrm{O}(\mathrm{O}) \mathrm{CH}), 168.16$ (s, $\mathrm{CH}=\mathrm{C}(\mathrm{N}) \mathrm{Ph}), 141.62,141.21,129.06,128.32 .128 .03 .127 .78$. 127.08 .126 .94 (s. $\left.\mathrm{C}_{6} \mathrm{H}_{5} \mathrm{C}(\mathrm{O}) \mathrm{CH}(\mathrm{N}) \mathrm{C}_{5} \mathrm{H}_{5}\right) .130 .72$ (s. $\mathrm{CH}_{2} \mathrm{C}$. $\left.\left(\mathrm{CH}_{2}\right) \mathrm{CH}_{2}\right) .96 .73$ (s. $\left.\mathrm{C}(\mathrm{O}) \mathrm{CH}=\mathrm{C}(\mathrm{N})\right) .70 .49$ (s. NCH, $\mathrm{CH}_{2}-$ $\left.\mathrm{CH}_{2} \mathrm{OCH} \mathrm{CH}_{2}\right) .60 .20$ (s. $\mathrm{NCH}_{2} \mathrm{CH}_{2} \mathrm{CH}_{2} \mathrm{OCH}_{3}$ ). 58.65 (s. $\mathrm{CH}_{2}$ of allyl), $58.48\left(\mathrm{~s}, \mathrm{CH}_{2}\right.$ of allyl). $53.56\left(\mathrm{~s} . \mathrm{N} \mathrm{CH}_{2} \mathrm{CH}_{2} \mathrm{CH}_{2} \mathrm{OCH}_{3}\right)$, 33.59 (s. $\mathrm{NCH}_{2} \mathrm{CH}_{2} \mathrm{CH}_{2} \mathrm{OCH}_{3}$ ). 23.81 (s. $\mathrm{CH}_{2}$ of allyl): Anal. Calcd. For $\mathrm{C}_{23} \mathrm{H}_{2}-\mathrm{NO}_{2} \mathrm{Pd}$ : C. $60.60: \mathrm{H}, 5.97: \mathrm{N}, 3.07$. Found: C. $60.55: \mathrm{H}, 6.20: \mathrm{N}, 2.84$.

$\mathrm{Pd}\left(\eta^{3}-\mathrm{CH}_{2} \mathrm{CMeCH}_{2}\right)\left(\mathrm{PhC}(\mathrm{O}) \mathrm{CHC}\left(\mathrm{NCH}_{2} \mathrm{CH}_{2} \mathrm{OCH}_{3}\right) \mathrm{Ph}\right)-$ (8a): y ield: 0.56g. 50\%. ${ }^{\mathrm{H}} \mathrm{H}-\mathrm{NMR}\left(199.976 \mathrm{MHz}, \mathrm{CDCl}_{3}\right): \delta$ $7.79 \sim 7.74,7.44 \sim 7.27\left(\mathrm{~m} . \quad 10 \mathrm{H} . \quad \mathrm{C}_{6} H_{5} \mathrm{C}(\mathrm{O}) \mathrm{CH}(\mathrm{N}) \mathrm{C}_{6} H_{5}\right)$. $5.41(\mathrm{~s}, 1 \mathrm{H}, \mathrm{C}(\mathrm{O}) \mathrm{CH}=\mathrm{C}(\mathrm{N})) .3 .79 \sim 3.71\left(\mathrm{~m}, 3 \mathrm{H}, \mathrm{NCH}_{2} \mathrm{CH}_{2}\right.$ $\mathrm{OCH}_{3}$, allỵl). 3.52(t. 2H. $\left.\mathrm{NCH}_{2} \mathrm{CH}_{2} \mathrm{OCH}_{3}\right), 3.2 \mathrm{l}(\mathrm{s}$. $3 \mathrm{H}$, $\left.\mathrm{NCH}_{2} \mathrm{CH}_{2} \mathrm{OC} H_{3}\right) .3 .13\left(\mathrm{~d}, 1 \mathrm{H} . \mathrm{J}_{\mathrm{HH}}=2.8 \mathrm{~Hz}\right.$, allyl), $3.08(\mathrm{~s}, 1 \mathrm{H}$. allỵl). 2.75 (s. $\mathrm{IH}$, allyl) $2.24\left(\mathrm{~s}, 3 \mathrm{H}, \mathrm{CH}_{3}\right.$ of allyl): ${ }^{13} \mathrm{C}-\mathrm{NMR}$ (50.289 MHz. $\mathrm{CDCl}_{2}$ ): $\delta 173.02$ (s. $\left.\mathrm{PhC}(\mathrm{O}) \mathrm{CH}\right) .169 .13$ (s, $\mathrm{CH}=C(\mathrm{~N}) \mathrm{Pl}), 1+1.54,141.04 .129 .18 .128 .70 .128 .34 .128 .06$, 127.88, 127.26, 127.12(s. $\left.\mathrm{C}_{6} H_{5} \mathrm{C}(\mathrm{O}) \mathrm{CH}(\mathrm{N}) \mathrm{C}_{6} H_{5}\right) .130 .83(\mathrm{~s}$. $\left.\mathrm{CH}_{2} \mathrm{C}\left(\mathrm{CH}_{3}\right) \mathrm{CH}_{2}\right), 96.87(\mathrm{~s}, \mathrm{C}(\mathrm{O}) \mathrm{CH}=\mathrm{C}(\mathrm{N})), 74.17\left(\mathrm{~s} . \mathrm{NCH}_{2}\right.$ $\left.\mathrm{CH}_{2} \mathrm{OCH} \mathrm{CH}_{3}\right), 60.32$ (s. $\mathrm{NCH}_{2} \mathrm{CH}_{2} \mathrm{OCH}_{3}$ ), 59.94(s. $\mathrm{CH}_{2}$ of allyl). $59.14\left(\mathrm{~s} . \mathrm{CH}_{2}\right.$ of allyl). $53.43\left(\mathrm{~s} . \mathrm{N} \mathrm{CH}_{2} \mathrm{CH}_{2} \mathrm{OCH}_{3}\right), 23.85(\mathrm{~s}$, $\mathrm{CH}_{3}$ of allyl): Anal. Calcd. For $\mathrm{C}_{2} \mathrm{H}_{25} \mathrm{NO}_{2} \mathrm{Pd}: \mathrm{C} .59 .80: \mathrm{H}$, 5.70: N. 3.17. Found: C. 59.93: H. 5.69: N. 2.98. 
$\mathrm{Pd}\left(\eta^{3}-\mathrm{CH}_{2} \mathrm{CMeCH}_{2}\right)\left(\mathrm{PhC}(\mathrm{O}) \mathrm{CHC}\left(\mathrm{NCH}_{2} \mathrm{C}_{6} \mathrm{H}_{4} \mathrm{OCH}_{3}\right) \mathrm{Ph}\right)$ (10a): vield: $1.02 \mathrm{~g} .80 \% .{ }^{1} \mathrm{H}-\mathrm{NMR}\left(199.976 \mathrm{MHz}, \mathrm{CDCl}_{3}\right)$ : $\tilde{\delta}$ $7.85 \sim 7.80,7.35-7.17\left(\mathrm{~m}, 11 \mathrm{H} . \mathrm{C}_{6} H_{5} \mathrm{C}(\mathrm{O}) \mathrm{CH}(\mathrm{N}) \mathrm{C}_{6} H_{5}, \mathrm{NCH}_{2}-\right.$ $\left.\mathrm{C}_{6} \mathrm{H}_{4} \mathrm{OCH}_{3}\right), 7.47\left(\mathrm{~d}, 1 \mathrm{H}, \mathrm{J}_{\mathrm{HH}}=7.6 \mathrm{~Hz} . \mathrm{NCH}_{2} \mathrm{C}_{6} H_{4} \mathrm{OCH}_{3}\right)$. $7.00\left(\right.$ t. $1 \mathrm{H} . \mathrm{J}_{\mathrm{HH}}=7.4 \mathrm{~Hz}$. $\mathrm{NCH}_{2} \mathrm{C}_{6} H_{4} \mathrm{OCH}_{3}$ ). $6.80\left(\right.$ d. $1 \mathrm{H} . \mathrm{J}_{\mathrm{HH}}=$ $\left.7.6 \mathrm{~Hz} . \mathrm{NCH}_{-} \mathrm{C}_{6} H_{4} \mathrm{OCH}_{3}\right), 5.53(\mathrm{~s} .1 \mathrm{H}, \mathrm{C}(\mathrm{O}) \mathrm{CH}=\mathrm{C}(\mathrm{N})) .4 .83$ (q. $2 \mathrm{H}, \mathrm{J}_{\mathrm{HH}}=16.4 \mathrm{~Hz}, \mathrm{NCH}_{3} \mathrm{C}_{6} \mathrm{H}_{4} \mathrm{OCH}_{3}$ ). 3.74 (s. $3 \mathrm{H}, \mathrm{NCH}_{3}-$ $\left.\left.\mathrm{C}_{4} \mathrm{H}_{4} \mathrm{OCH}\right)_{3}\right), 3.62\left(\mathrm{~d}, 1 \mathrm{H}, \mathrm{J}_{\mathrm{HH}}=2.8 \mathrm{~Hz}\right.$, allyl $), 2.88(\mathrm{~s}, 1 \mathrm{H}$. allyl) $2.52\left(\mathrm{~d} .1 \mathrm{H}, \mathrm{J}_{\mathrm{HH}}=2.6 \mathrm{~Hz}\right.$, allyl) 2.32 (s. $1 \mathrm{H}$. allyl), 1.80 (s. $3 \mathrm{H} . \mathrm{CH}_{3}$ of allyl). ${ }^{13} \mathrm{C}-\mathrm{NMR}\left(50.289 \mathrm{MHz}, \mathrm{CDCl}_{3}\right)$ : 173.08(s. $\mathrm{PhC}(\mathrm{O}) \mathrm{CH}), \quad 169.10(\mathrm{~s}, \mathrm{CH}=\mathrm{C}(\mathrm{N}) \mathrm{Ph}) . \quad 155.79$. 141.65 . 140.72. 130.73, 129.17, 128.30, 128.09, 127.92. 127.17. 126.45. 120.56, 109.46(s. $\mathrm{C}_{6} \mathrm{H}_{5} \mathrm{C}(\mathrm{O}) \mathrm{CH}(\mathrm{N}) \mathrm{C}_{6} \mathrm{H}_{s}$ $\mathrm{NCH}_{2} \mathrm{C}_{6} \mathrm{H}_{4} \quad \mathrm{OCH}_{3}$ ). 130.91(s. $\left.\mathrm{CH}_{2} \mathrm{C}\left(\mathrm{CH}_{3}\right) \mathrm{CH}_{2}\right) .96 .69$ (s. $\mathrm{C}(\mathrm{O}) \mathrm{CH}=\mathrm{C}(\mathrm{N})) .59 .20\left(5, \mathrm{NCH}_{2} \mathrm{C}_{6} \mathrm{H}_{4} \mathrm{OCH}_{3}\right) .59 .01\left(\mathrm{~s} . \mathrm{CH}_{2}\right.$ of allyl), $55.36\left(\mathrm{~s} . \mathrm{CH}_{2}\right.$ of allyl). $54.64\left(\mathrm{~s}, \mathrm{NCH}_{2} \mathrm{C}_{6} \mathrm{H}_{4} \mathrm{OCH}_{3}\right)$. 23.17(s. $\mathrm{CH}_{3}$ of allyl): Anal. Calcd. For $\mathrm{C}_{2} \mathrm{H}_{2}: \mathrm{NO}_{2} \mathrm{Pd}$ : C. 64.35: H. 5.40: N, 2.78. Found: C. 64.55; H. 5.55; N. 2.52.

$\mathrm{Pd}\left(\eta^{3}-\mathrm{CH}_{2} \mathrm{CMeCH}_{2}\right)\left(\mathrm{PhC}(\mathrm{O}) \mathrm{CHC}\left(\left(\mathrm{NCH}_{2} \mathrm{CH}_{2} \mathrm{C}_{5} \mathrm{H}_{4} \mathrm{~N}\right) \mathrm{Ph}\right)\right.$ $\left(\mathbf{C}_{5} \mathrm{H}_{4} \mathbf{N}=\mathbf{2 - p y r i d y l}\right)(\mathbf{1 2 a})$ : yield: $0.37 \mathrm{~g}, 30 \% .{ }^{1} \mathrm{H}-\mathrm{NMR}(400$ $\left.\mathrm{MHz} . \mathrm{CDCl}_{3}\right): \delta 8.44 .7 .80 \sim 7.75 .7 .51 \sim 7.02 .6 .89(\mathrm{~m} .13 \mathrm{H}$. $\mathrm{C}_{6} H_{5} \mathrm{C}(\mathrm{O}) \mathrm{CH}(\mathrm{N}) \mathrm{C}_{6} H_{5 .} \mathrm{C} H$ of Pyridine). 5.38 (s. $\mathrm{IH} . \mathrm{C}(\mathrm{O}) \mathrm{CH}=$ $\mathrm{C}(\mathrm{N})), 3.89\left(\mathrm{t} .2 \mathrm{H} . \mathrm{J}_{\mathrm{HH}}=7.0 \mathrm{~Hz}, \mathrm{NCH}_{z} \mathrm{CH}_{-} \mathrm{Py}\right.$ ridine). 3.79 (br s. $\mathrm{lH}$. allyl). 3.39 (br s. $\mathrm{lH}$, allyl) 3.06 (br t. $3 \mathrm{H}, \mathrm{NCH}_{2} \mathrm{CH}_{3-}$ Pyridine, allyl). 2.83 (brs. $1 \mathrm{H}$, allyl). 2.27 (s, $3 \mathrm{H} . \mathrm{CH}_{3}$ of allyl); Anal. Calcd. For $\mathrm{C}_{26} \mathrm{H}_{26} \mathrm{~N}_{2} \mathrm{OPd}$ C. 63.87: H. 5.36: N. 5.73 . Found: C. 63.96: H. 5.62: N, 5.42.

$\mathrm{Pd}\left(\mathrm{CH}_{3} \mathrm{C}(\mathrm{O}) \mathrm{CHC}\left(\left(\mathrm{NCH}_{2} \mathrm{CH}_{2} \mathrm{C}_{6} \mathrm{H}_{4} \mathrm{~N}\right) \mathrm{CH}_{3}\right)\left(\mathrm{PPh}_{3}\right) \mathrm{Me}\right.$ $\left(\mathrm{C}_{3} \mathrm{H}_{4} \mathrm{~N}=\right.$ 2-pyridyl) (6b): Ligand $\mathrm{CH}_{3} \mathrm{C}(\mathrm{O}) \mathrm{CHC}\left(\left(\mathrm{NCH}_{2} \mathrm{CH}_{2}-\right.\right.$ $\left.\mathrm{C}_{3} \mathrm{H}_{4} \mathrm{~N}\right) \mathrm{CH}_{3}(0.20 \mathrm{~g} .1 .0 \mathrm{mmol})$ was dissolved in $20 \mathrm{~mL}$ of THF and this solution was added to a mixture of distilled THF $(30 \mathrm{~mL})$ and $\mathrm{Tl}\left(\mathrm{OC}_{2} \mathrm{H}_{5}\right)(0.30 \mathrm{~g} .1 .20 \mathrm{mmol})$ dropwise. The resulting solution was stirred at room temperature for $1 \mathrm{hr}$ and then the solvent was removed in vacuum. $30 \mathrm{~mL}$ of THF was added and $\left[\mathrm{Pd}\left(\mathrm{PPl}_{3}\right) \mathrm{MeCl}\right]_{2}(0.51 \mathrm{~g} .0 .60 \mathrm{mmol})$ in $70 \mathrm{~mL}$ of THF was added to this solution dropwise. After stirring at room temperature for $8 \mathrm{hr}$. the mixture was filtered through celite on a frit into a Schlenk flask and then the solvent was removed in vacuum. The product was washed with distilled hexane and extracted with methylene chloride. The solvent was removed in vacuum to give a green solid. (yield: $0.25 \mathrm{~g}$. $43 \%$ ) ${ }^{1} \mathrm{H}-\mathrm{NMR}\left(199.976 \mathrm{MHz}, \mathrm{CDCl}_{3}\right): \delta 8.54(\mathrm{~d} .1 \mathrm{H} . \mathrm{J}=$ $6.2 \mathrm{~Hz}) .7 .68-7.05(\mathrm{~m} .18 \mathrm{H})(\mathrm{PPh}$ and $P y) .4 .72(\mathrm{~s} .1 \mathrm{H} . \mathrm{C}(\mathrm{O})$ $\mathrm{CH}=\mathrm{C}(\mathrm{N})), 3.88\left(\mathrm{~m} .2 \mathrm{H}, \mathrm{NCH} \mathrm{CH}_{2} \mathrm{Py}\right) .3 .10(\mathrm{dt}, 2 \mathrm{H} . \mathrm{J}=$ 7.8.7.4Hz, $\mathrm{NCH}_{2} \mathrm{CH}_{2} \mathrm{Py}$ ), $1.98\left(\mathrm{~s}, 3 \mathrm{H}, \mathrm{C}(\mathrm{N}) \mathrm{CH}_{3}\right), 1.85$ (s. $3 \mathrm{H}$. $\mathrm{CH}_{3} \mathrm{C}(\mathrm{O})$ ). $0.2 \mathrm{l}\left(\mathrm{d} . \mathrm{J}=3.2 \mathrm{~Hz}, 3 \mathrm{H} . \mathrm{CH}_{3}\right):{ }^{13} \mathrm{C}-\mathrm{NMR}(50.289$ $\left.\mathrm{MHz} . \mathrm{CDCl}_{3}\right): \delta 179.00\left(\mathrm{CH}_{3} \mathrm{C}(\mathrm{O}) \mathrm{CH}\right), 156.68\left(\mathrm{CH}=\mathrm{C}(\mathrm{N}) \mathrm{CH}_{3}\right)$, $152.98,135.05,134.98,134.87,134.76,130.05,128 .+2$. 128.11. 127.95. 127.85, 127.07, 126.61. 121.45. 109.53(PPh and $\mathrm{Py}), 97.56(\mathrm{C}(\mathrm{O}) \mathrm{CH}=\mathrm{C}(\mathrm{N})), 40.74\left(\mathrm{NCH}_{2} \mathrm{CH}_{3}\right), 31.88$ $\left(\mathrm{NCH}_{2} \mathrm{CH}_{2}\right) \cdot 23.84\left(\mathrm{CH}_{3} \mathrm{C}(\mathrm{O}) \mathrm{CH}\right) .16 .82\left(\mathrm{CH}=\mathrm{C}\left(\mathrm{N}^{2} \mathrm{CH}_{3}\right) .0 .819\right.$ $\left(\mathrm{CH}_{3}\right) ;{ }^{31} \mathrm{P}-\mathrm{NMR}\left(162.027 \mathrm{MHz}, \mathrm{CDCl}_{3}\right): \delta$ 44.77; Anal. Calcd. For $\mathrm{C}_{31} \mathrm{H}_{33} \mathrm{~N}_{2} \mathrm{OPPd}: \mathrm{C}$. 63.43: H. 5.71: N. 4.77: Found C. $63.56 ;$ H. $5.95 ;$ N. 4.86 .

$\mathrm{Pd}\left(\mathrm{PhC}(\mathrm{O}) \mathrm{CHC}\left(\left(\mathrm{NCH}_{2} \mathrm{CH}_{2} \mathrm{C}_{3} \mathrm{H}_{4} \mathrm{~N}\right) \mathrm{Ph}\right)\left(\mathrm{PPh}_{3}\right) \mathrm{Me}\left(\mathrm{C}_{3} \mathrm{H}_{4} \mathrm{~N}\right.\right.$ = 2-pyridyl) (12b): yield: 0.58 g. $81 \% .{ }^{1} \mathrm{H}-\mathrm{NMR}$ (199.976 $\left.\mathrm{MHz}, \mathrm{CDCl}_{3}\right): \delta 8.42($ d. $1 \mathrm{H} . \mathrm{J}=6.2 \mathrm{~Hz}) .7 .77-6.99(\mathrm{~m} .28 \mathrm{H})$ $\left(\mathrm{PPh}_{3} . P h\right.$ and $\left.P y\right) .5 .37(\mathrm{~s} .1 \mathrm{H} . \mathrm{C}(\mathrm{O}) \mathrm{CH}=\mathrm{C}(\mathrm{N})) .3 .80(\mathrm{~m} .2 \mathrm{H}$.
$\left.\mathrm{NCH}_{2} \mathrm{CH}_{2} \mathrm{Py}\right) .3 .16\left(\mathrm{t}, 2 \mathrm{H}_{3} \mathrm{~J}=15.6 \mathrm{~Hz} . \mathrm{NCH}_{2} \mathrm{CH} H_{2} \mathrm{Py}\right), 0.35(\mathrm{~d}$, $\left.\mathrm{J}=3.0 \mathrm{~Hz}, 3 \mathrm{H}, \mathrm{CH}_{3}\right):{ }^{13} \mathrm{C}-\mathrm{NMR}\left(50.289 \mathrm{MHz}, \mathrm{CDCl}_{3}\right): \delta$ $172.54(\mathrm{PlC}(\mathrm{O}) \mathrm{CH}), 160.15(\mathrm{CH}=\mathrm{C}(\mathrm{N}) \mathrm{Ph}), 1+2.36 .1+2.69$, 142.64. 140.88. 136.09, 135.04, 134.93. 131.87, 131.40, 130.29. 130.27. 128.76, 128.48, 128.38. 128.27, 127.68. 127.41, 127.21. 127.16, 123.64, 121.17 ( $\mathrm{PPh}_{3} . \mathrm{Ph}$ and $\left.P y\right)$, $96.52(\mathrm{C}(\mathrm{O}) \mathrm{CH}=\mathrm{C}(\mathrm{N})), 52.04\left(\mathrm{NCH}_{2} \mathrm{CH}_{2}\right), 42.88\left(\mathrm{NCH}_{2}\left(\mathrm{CH}_{2}\right)\right.$. $0.92\left(\mathrm{CH}_{3}\right):{ }^{31} \mathrm{P}-\mathrm{NMR}\left(162.027 \mathrm{MHz} . \mathrm{CDCl}_{3}\right): \delta 43.73$; Anal. Calcd. For $\mathrm{C}_{41} \mathrm{H}_{3}: \mathrm{N}_{2}$ OPPd: $\mathrm{C}$. 69.25: H. 5.24: N. 3.94: Found C. $69.33: \mathrm{H}, 5.16: \mathrm{N}, 3.74$.

$\mathrm{Pd}\left(\mathrm{CF}_{3} \mathrm{C}(\mathrm{O}) \mathrm{CHC}\left(\mathrm{NCH}_{2} \mathrm{CH}_{2} \mathrm{CH}_{2} \mathrm{OCH}_{3}\right) \mathrm{CF}_{3}\right)\left(\mathrm{PPh}_{3}\right) \mathrm{Me}$ (13b): yield: 0.56 g. $85 \% .{ }^{1} \mathrm{H}-\mathrm{NMR}\left(199.976 \mathrm{MHz}, \mathrm{CDCl}_{3}\right)$ : $\tilde{\mathrm{d}}$ $7.70-7.36\left(\mathrm{~m} .15 \mathrm{H}, \mathrm{PPh}_{3}\right), 5.581$ (s. $\left.1 \mathrm{H}, \mathrm{C}(\mathrm{O}) \mathrm{CH}=\mathrm{C}(\mathrm{N})\right)$, 3.811 (br. $2 \mathrm{H} . \mathrm{NCH} \mathrm{CH}_{2}$ ) .3 .438 (t. $2 \mathrm{H} . \mathrm{J}=8.6 \mathrm{~Hz}_{2} \mathrm{CH}_{2} \mathrm{CH}_{2}$ ) $\mathrm{OMe}$ ), $3.320\left(\mathrm{~s} .3 \mathrm{H} . \mathrm{OCH}_{3}\right)$. 2.127 (m. $2 \mathrm{H}$. $\mathrm{NCH}_{2} \mathrm{CH}_{2} \mathrm{CH}_{2}$ ), $0.278\left(\mathrm{~d}, 3 \mathrm{H} . \mathrm{J}=2.8 \mathrm{~Hz} . \mathrm{CH}_{3}\right) ;{ }^{12} \mathrm{C}-\mathrm{NMR}\left(50.289 \mathrm{MHz} . \mathrm{CDCl}_{3}\right)$ : o $165.99\left(\mathrm{CF}_{3} \mathrm{C}(\mathrm{O}) \mathrm{CH}\right) .154 .90\left(\mathrm{CH}=\mathrm{C}(\mathrm{N}) \mathrm{CF}_{3}\right), 135.05 \sim 128.05$ $\left(\mathrm{PPh}_{3}\right) .120 .75,117.90\left(\mathrm{CF}_{3}\right) .87 .35(\mathrm{C}(\mathrm{O}) \mathrm{CH}=\mathrm{C}(\mathrm{N})) .70 .27$ $\left(\mathrm{NCH}_{2} \mathrm{CH}_{2}\right), 58.56\left(\mathrm{CH}_{2} \mathrm{CH}_{2} \mathrm{OMe}\right) .49 .18\left(\mathrm{OCH}_{3}\right) .34 .29\left(\mathrm{NCH}_{2}-\right.$ $\left.\mathrm{CH}_{2} \mathrm{CH}_{2}\right) .0 .1+\left(\mathrm{CH}_{3}\right):{ }^{21} \mathrm{P}-\mathrm{NMR}\left(162.027 \mathrm{MHz}, \mathrm{CDCl}_{3}\right): \delta$ 42.99: Anal. Calcd. For $\mathrm{C}_{2} \mathrm{H}_{8} \mathrm{~F}_{6} \mathrm{NO}_{-} \mathrm{PPd}$ : C. 50.81: H. 4.26: N. 2.12: Found: C. 51.23: H. 4.46: N. 1.65 .

$\mathrm{Pd}\left(\mathrm{CH}_{3} \mathrm{C}(\mathrm{O}) \mathrm{CHC}\left(\mathrm{NCH}_{2} \mathrm{CH}_{2} \mathrm{C}_{5} \mathrm{H}_{4} \mathrm{~N}\right) \mathrm{CH}_{3}\right) \mathrm{Me}\left(\mathrm{C}_{5} \mathrm{H}_{4} \mathrm{~N}=\right.$ 2-pyridyl) (6c): Ligand $\mathrm{MeC}(\mathrm{O}) \mathrm{CHC}\left(\left(2-\mathrm{NHCH}_{2} \mathrm{CH}_{2}\right) \mathrm{Py}\right.$ ridine) Me( $0.25 \mathrm{~g}, 1.22 \mathrm{mmol})$ was dissolved in $20 \mathrm{ml}$ of THF and this solution was added to a mixture of distilled THF ( 30 $\mathrm{mL})$ and $\mathrm{Tl}\left(\mathrm{OC}_{2} \mathrm{H}_{5}\right)(0.30 \mathrm{~g}$. $1.20 \mathrm{mmol})$ dropwise. The resulting solution was stirred at room tenperature for llur and then the solvent was removed in vacuum. $30 \mathrm{~mL}$ of THF was added and $\mathrm{Pd}\left(\mathrm{NH}_{2} \mathrm{CH}_{2} \mathrm{C}_{6} \mathrm{H}_{5}\right)=\mathrm{MeCl}(0.45 \mathrm{~g}, 1.20 \mathrm{mmol})$ in $70 \mathrm{~mL}$ of THF was added to this solution dropwise. After stirring at room tenperature for $8 \mathrm{lur}$. the mixture was filtered through celite on a frit into a Schlenk flask and then the solvent was removed in vacuum. The product was washed with distilled hexane and extracted with methylene clloride. The solvent was removed in vacuum. (yield: $0.21 \mathrm{~g} .52 \%$ )

${ }^{l} \mathrm{H}-\mathrm{NMR}\left(199.976 \mathrm{MHz} . \mathrm{CDCl}_{3}\right): \hat{o} 8.53$ (d. $\left.1 \mathrm{H} . \mathrm{J}=2.6 \mathrm{~Hz}\right)$, $7.71(\mathrm{dt} . \mathrm{lH}, \mathrm{J}=0.8 .8 .6 \mathrm{~Hz}) .7 .32-7.27(\mathrm{~m}, \mathrm{lH}) .7 .16-7.12(\mathrm{dt}$. lH. $\mathrm{J}=0.6,7 .+\mathrm{Hz})(P \mathrm{v}) .4 .78(\mathrm{~s} .1 \mathrm{H}, \mathrm{C}(\mathrm{O}) \mathrm{CH}=\mathrm{C}(\mathrm{N})) .3 .29$ (br. $4 \mathrm{H}, \mathrm{NCH}, \mathrm{CH}, \mathrm{Py}), 1.94\left(\mathrm{~s}, 3 \mathrm{H} . \mathrm{C}(\mathrm{N}) \mathrm{CH}_{3}\right) .1 .82(\mathrm{~s}, 3 \mathrm{H}$, $\left.\mathrm{CH}_{3} \mathrm{C}(\mathrm{O})\right), 0.62$ (s. $\left.3 \mathrm{H} . \mathrm{CH}_{3}\right):{ }^{13} \mathrm{C}-\mathrm{NMR}\left(50.289 \mathrm{MHz}, \mathrm{CDCl}_{3}\right)$ : ô $175.78\left(\mathrm{CH}_{3} \mathrm{C}(\mathrm{O}) \mathrm{CH}\right) .163 .83\left(\mathrm{CH}=\mathrm{C}(\mathrm{N}) \mathrm{CH}_{3}\right) .161 .65 .153 .22$. 137.92. 124.17. $122.95(P v) .98 .04(\mathrm{C}(\mathrm{O}) \mathrm{CH}=\mathrm{C}(\mathrm{N})) .46 .58$ $\left(\mathrm{NCH}_{2} \mathrm{CH}_{2}\right) .41 .75\left(\mathrm{NCH}_{2}\left(\mathrm{CH}_{2}\right), 26.67\left(\mathrm{CH}_{3} \mathrm{C}(\mathrm{O}) \mathrm{CH}\right), 22.43\right.$ $\left(\mathrm{CH}=\mathrm{C}(\mathrm{N}) \mathrm{CH}_{3}\right) \cdot 3.74\left(\mathrm{CH}_{3}\right)$ : Anal. Calcd. For $\mathrm{C}_{13} \mathrm{H}_{18} \mathrm{~N}_{2} \mathrm{OPd}$ : C. 48.09: H. 5.59: N. 8.63. Found: C. 48.24: H. 5.37: N. 8.65 .

$\mathrm{Pd}\left(\mathrm{PhC}(\mathrm{O}) \mathrm{CHC}\left(\left(\mathrm{NCH}_{2} \mathrm{CH}_{2} \mathrm{C}_{5} \mathrm{H}_{4} \mathrm{~N}\right) \mathrm{Ph}\right) \mathrm{Me}\left(\mathrm{C}_{5} \mathrm{H}_{4} \mathrm{~N}=\mathbf{2 -}\right.\right.$ pyidyl) (12c): yield: 0.19 g. 49\%. ${ }^{\mathrm{H}} \mathrm{H}-\mathrm{NMR}(199.976 \mathrm{MHz}$. $\left.\mathrm{CDCl}_{3}\right): \hat{o} 8.66(\mathrm{~d} .1 \mathrm{H}, \mathrm{J}=5.4 \mathrm{~Hz}), 7.83(\mathrm{~m} 2 \mathrm{H}), 7.73(\mathrm{t} 1 \mathrm{H} . \mathrm{J}=$ $7.6 \mathrm{~Hz}) .7 .37-7.20(\mathrm{~m}, 10 \mathrm{H}) .(P h) .5 .52(\mathrm{~s} . \mathrm{lH} . \mathrm{C}(\mathrm{O}) \mathrm{CH}=$ $\mathrm{C}(\mathrm{N})) .3 .21\left(\mathrm{br}, 4 \mathrm{H} . \mathrm{NC} H_{2} \mathrm{CH}_{2} \mathrm{Py}\right.$ ), 0.87(s. 3H. CH$\left.H_{3}\right){ }^{13} \mathrm{C} \cdot \mathrm{NMR}$ $\left(50.289 \mathrm{MHz} . \mathrm{CDCl}_{3}\right): \delta 171.78(\mathrm{PhC}(\mathrm{O}) \mathrm{CH}), 162.18(\mathrm{CH}=$ $C(\mathrm{~N}) \mathrm{Ph}), 153.47,141.84 .140 .99,138.05,129.03 .128 .54$. 128.34. 128.03, 127.19. 127.09. 124.31, 123.06(Ph). 96.40 $(\mathrm{C}(\mathrm{O}) \mathrm{CH}=\mathrm{C}(\mathrm{N})), 49.16\left(\mathrm{NCH}_{2} \mathrm{CH}_{2}\right), 42.28\left(\mathrm{NCH}_{2} \mathrm{CH}_{2}\right), 5.28$ $\left(\mathrm{CH}_{3}\right)$; Anal. Calcd. For $\mathrm{C}_{23} \mathrm{H}_{2} \mathrm{~N}_{2} \mathrm{OPd}: \mathrm{C}, 61.55: \mathrm{H}, 4.94: \mathrm{N}$, 6.24. Found: C. 61.89: H. 5.48: N. 6.31.

$\mathrm{Pd}\left(\mathrm{CH}_{3} \mathrm{C}(\mathrm{O}) \mathrm{CHC}\left(\left(\mathrm{NCH}_{2} \mathrm{C}_{5} \mathrm{H}_{4} \mathrm{~N}\right) \mathrm{CH}_{3}\right) \mathrm{Me}\left(\mathrm{C}_{5} \mathrm{H}_{4} \mathrm{~N}=2\right.\right.$ 2-pyi- 
dyl) (14c): yield: $0.29 \mathrm{~g} .61 \%{ }^{\mathrm{l}} \mathrm{H}-\mathrm{NMR}(199.976 \mathrm{MHz}$. $\left.\mathrm{CDCl}_{3}\right): \delta 8.48(\mathrm{~d}, \mathrm{lH}, \mathrm{J}=5.8 \mathrm{~Hz}) .7 .76(\mathrm{dt} .1 \mathrm{H} . \mathrm{J}=0.8 .8 .6 \mathrm{~Hz})$. $7.389 \sim 7.190(\mathrm{~m}, 2 \mathrm{H}) .(P v), 4.95(\mathrm{~s}, 1 \mathrm{H} . \mathrm{C}(\mathrm{O}) \mathrm{CH}=\mathrm{C}(\mathrm{N})) .4 .89$ $\left(\mathrm{s}, 2 \mathrm{H}, \mathrm{NCH}_{2}\right) .2 .04\left(\mathrm{~s} .3 \mathrm{H} . \mathrm{C}(\mathrm{N}) \mathrm{CH}_{3}\right) .1 .95\left(\mathrm{~s} .3 \mathrm{H} . \mathrm{CH} \mathrm{C}_{3} \mathrm{C}(\mathrm{O})\right.$ ). $0.6+$ (s. 3H. CH $\left.\mathrm{CH}_{3}\right){ }^{13} \mathrm{C}-\mathrm{NMR}\left(50.289 \mathrm{MHz}, \mathrm{CDCl}_{3}\right):$ ò 176.72 $\left(\mathrm{CH}_{3} \mathrm{C}(\mathrm{O}) \mathrm{CH}\right), 165.41\left(\mathrm{CH}=\mathrm{C}(\mathrm{N}) \mathrm{CH}_{3}\right) .162 .05,149.42,136.83$. $123.15,121.48(P v), 98.52(\mathrm{C}(\mathrm{O}) \mathrm{CH}=\mathrm{C}(\mathrm{N})), 58.98\left(\mathrm{NCH}_{2}\right)$. $27.00\left(\mathrm{CH}_{3} \mathrm{C}(\mathrm{O}) \mathrm{CH}\right) .20 .80\left(\mathrm{CH}=\mathrm{C}(\mathrm{N}) \mathrm{CH}_{3}\right) .1 .54\left(\mathrm{CH}_{3}\right)$ : Anal. Calcd. For $\mathrm{C}_{12} \mathrm{H}_{16} \mathrm{~N}_{2} \mathrm{OPd}$ : C. 46.39: H. 5.19: N. 9.02: Found C. $46.50:$ H. $5.53:$ N. 8.61 .

$\mathrm{Pd}\left(\mathrm{PhC}(\mathrm{O}) \mathrm{CHC}\left(\left(\mathrm{NCH}_{2} \mathrm{C}_{5} \mathrm{H}_{4} \mathrm{~N}\right) \mathrm{Ph}\right) \mathrm{Me}\left(\mathrm{C}_{5} \mathrm{H}_{4} \mathrm{~N}=2\right.\right.$ - pyqridyl) (15c): yield: $0.22 \mathrm{~g}, 55 \% .{ }^{\mathrm{j}} \mathrm{H}-\mathrm{NMR}\left(199.976 \mathrm{MHz}^{\mathrm{CDCl}} \mathrm{CDC}_{3}\right)$. ô 8.31 (dt. $1 \mathrm{H} . \mathrm{J}=6.4 \mathrm{~Hz}), 7.86(\mathrm{dd} \mathrm{lH} . \mathrm{J}=0.8,4.8 \mathrm{~Hz}), 7.81$ (dd $\mathrm{IH} . \mathrm{J}=0.8+4.8 \mathrm{~Hz}) .7 .38-7.10(\mathrm{~m} .10 \mathrm{H}) .7 .16(\mathrm{t} . \mathrm{IH} . \mathrm{J}=7.8 \mathrm{~Hz})$ $(P h$ and $P V) .5 .58(5,1 \mathrm{H}, \mathrm{C}(\mathrm{O}) \mathrm{CH}=\mathrm{C}(\mathrm{N})), 4.81\left(\mathrm{~s} .2 \mathrm{H} . \mathrm{NCH}_{2}\right)$. $0.88\left(\mathrm{~s} .3 \mathrm{H}, \mathrm{CH}\right.$ ) $;{ }^{13} \mathrm{C}-\mathrm{NMR}\left(50.289 \mathrm{MHz} . \mathrm{CDCl}_{3}\right)$ : ô 175.06 $(\mathrm{PhC}(\mathrm{O}) \mathrm{CH}), 164.29(\mathrm{CH}=\mathrm{C}(\mathrm{N}) \mathrm{Ph}), 162.71,161.16,150.05$. $148.97,141.17 .138 .95,137.70,136.72 .130 .03,128.94 .128 .43$, 127.99. 127.20,125.41. 124.09, 120.78 ( $P h$ and $\left.P_{y}\right), 96.40$ $(\mathrm{C}(\mathrm{O}) \mathrm{CH}=\mathrm{C}(\mathrm{N})) .44 .28\left(\mathrm{NCH}_{2}\right) .5 .28\left(\mathrm{CH}_{3}\right)$; Anal. Calcd. For $\mathrm{C}_{22} \mathrm{H}_{20} \mathrm{~N}_{2} \mathrm{OPd}$ : C. 60.77: H. 4.64: N. 6.44: Found C. 60.43: H. $4.60:$ N. 6.34 .

$\mathbf{P d}\left(\mathrm{PhCH}_{2} \mathrm{NH}_{3}\right)_{2} \mathrm{MeCl}(\mathbf{1 6}): \mathrm{Pd}(\mathrm{COD}) \mathrm{MeCl}(1.00$ g. 3.77 mmol) was dissolved in $20 \mathrm{~mL}$ of $\mathrm{CH}_{3} \mathrm{CN}$ and this solution was added to a mixture of distilled THF $(30 \mathrm{~mL})$ and $\mathrm{C}_{6} \mathrm{H}_{5-}$ $\mathrm{CH}_{2} \mathrm{NH}_{2} 0.81 \mathrm{~g}(7.58 \mathrm{mmol})$ dropwise. After stirring at room temperature for $30 \mathrm{~min}$, the mixture was filtered and then the product was removed in vacuum. The product was washed with distilled $\mathrm{CH}_{3} \mathrm{CN}$. The solvent was removed in vacuum to give a white solid. (yield: $1.35 \mathrm{~g}, 95 \%$ )

${ }^{1} \mathrm{H}-\mathrm{NMR}\left(199.976 \mathrm{MHz}, \mathrm{CDCl}_{3}\right): \vec{\delta} 7.3+-7.27(\mathrm{~m}, 10 \mathrm{H})$. $3.99(\mathrm{dt}, 4 \mathrm{H}, \mathrm{J}=2.2,15 \mathrm{~Hz}), 2.75(\mathrm{br}, 4 \mathrm{H}), 0.40(\mathrm{~s}, 3 \mathrm{H})$ : ${ }^{13} \mathrm{C}-\mathrm{NMR}\left(50.289 \mathrm{MHz}, \mathrm{CDCl}_{3}\right)$ : ò 139.21, 129.21, 128.39 . $128.3 \mathrm{l}(\mathrm{Ph}) .49 .80\left(\mathrm{PhCH}_{2}\right),-3.91\left(\mathrm{CH}_{3}\right)$ : Anal. Calcd. For $\mathrm{C}_{15} \mathrm{H}_{21} \mathrm{ClN} \_\mathrm{Pd}$ : C. 48.53 ; H. 5.70; N. 7.55. Found: C, 48.67 : H. 6.16: N. 7.52, Mp. $130^{\circ} \mathrm{C}$ dec. white solid

Homopolymerization of norbomene with catalysts acti-

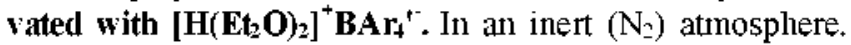
$\left[\mathrm{H}\left(\mathrm{Et}_{2} \mathrm{O}\right)_{3}\right] \mathrm{BAr}_{4}{ }^{-}$(catalyst : cocatalyst $=1: 1$ ) were placed into a small vial. To this was added approximately $5 \mathrm{~mL}$ of solvent, and the mixture gently shaken to dissolve the solids. This solution was then added to $0.005 \mathrm{~g}$ of catalyst, 1000 equivalents of norbornene dissolved in approximately $10 \mathrm{~mL}$ of solvent in a $20 \mathrm{~mL}$ vial containing a stir bar. The solution was stirred rapidly for about $5 \mathrm{~min}$ after which time polymerization was quenched by adding acidified methanol. The solid was washed with methanol and dried under vacuum. The resultant polymer was found to be insoluble in all common laboratory solvents.

Homopolymerization of norbomene with catalysts activated with MMAO. In an inert $\left(\mathrm{N}_{z}\right)$ atmosphere. $\mathrm{MMAO}$ (catalyst : cocatalyst $=1: 1000$ ) were placed into a small vial. To this was added approximately $5 \mathrm{~mL}$ of solvent. and the mixture gently shaken to dissolve the solids. This stock solution was then added to $0.005 \mathrm{~g}$ of catalyst. 1000 equivalent of norbomene dissolved in approximately $10 \mathrm{~mL}$ of solvent in a $20 \mathrm{~mL}$ vial containing a stirring bar. The solution was stirred rapidly for about $2 \mathrm{~min}$. after which time poly merization was complete. The reaction was quenched by addition of acidified methanol. The solid was washed with methanol and dried under vacuum.

Structural detemination. The cry stals suitable for the X-ray crystallography were grown from the methylene chloride solutions in the freezer maintained at $-20^{\circ} \mathrm{C}$.

The diffraction data for compounds $1 \mathrm{a}, 4 \mathrm{~b}$, and $12 \mathrm{c}$ were collected on a Bruker CCD diffractoneter with $\mathrm{MoK}_{u}(\lambda=$ 0.71073 ) radition employing $2 \mathrm{~kW}$ sealed tube $\mathrm{X}$-ray source operating at $1.6 \mathrm{~kW}$. The crystals were mounted on glass fibers under eposy. The reflections were successfully indexed by an automated indexing routine built in the SMART program (SMART Area-Detector Software Package. Bruker AXS, Inc.. Madison WI. 1995). The CCD data were integrated and scaled using a Bruker SAINT (SAX Area-Detector Integration Program, version 4.050, Bruker AXS. Inc., Madison WI, 1995). and the structures were solved and refined using XPREP (part of the SHELXTL. Crystal Structure Determination Package, version 5.04. Bnuker AXS. Inc. Madison WI, 1995) and SHELX-97 (Sheldrick. G. M. Institut fur Anorganische chemie der Universitat. Gotingen. F. R. G.. 1997). Hydrogen atoms were located in the calculated positions.

The crystallograplic data for compounds la and 12c (CCDC 608594,608596 ) are listed in Table $\mathrm{L}^{23}$ (The crystal structure and crystallographic data for compound th (CCDC 608595) was published elsewhere."

\section{Results and Discussion}

Ligand synthesis. Most of the functional $\beta$-ketoimines used in this study have been prepared by condendation reactions between corresponding $\beta$-diketones and amines described in the literature (scheme 1) with moderate to good yields. However, some of them were synthesized wa TMS intermediates as shown in scheme 2 and lower yields were generally observed due to multistep reactions

In all the $\beta$-ketoimines. enamine forms are predominant and the proton on the $\mathrm{N}$ atom couples with a protons if any.

Complex synthesis. Three classes of palladium(II) complexes have been prepared to investigate the ligand effect on the structure of the complex and the polymerization activity. These complexes are originally designed to have the formula of $\left[\mathrm{Pd}\left(\mathrm{N}^{\wedge} \mathrm{O}^{\wedge} \mathrm{L}\right) \mathrm{R}\right]$. which resembles the well-known salicylaldiminato complexes but $\mathrm{L}$ is incorporated into the $\left(\mathrm{N}^{\wedge} \mathrm{O}\right)$ ligand $\left(\mathrm{N}^{\wedge} \mathrm{O}\right.$ ligand with an internal base). The target conplexes

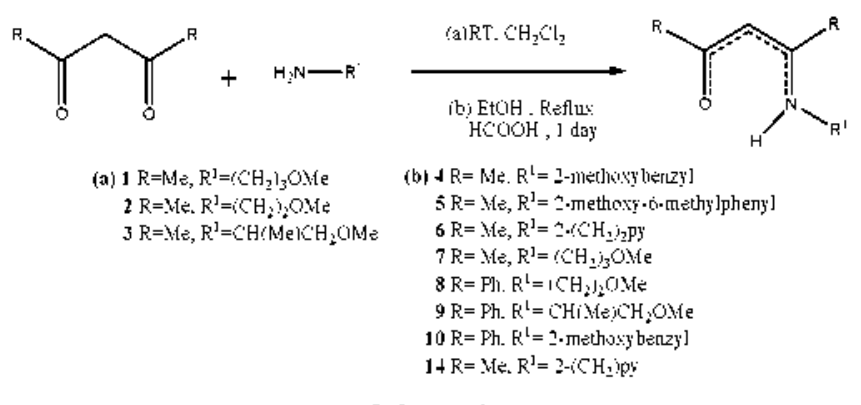

Scheme 1 


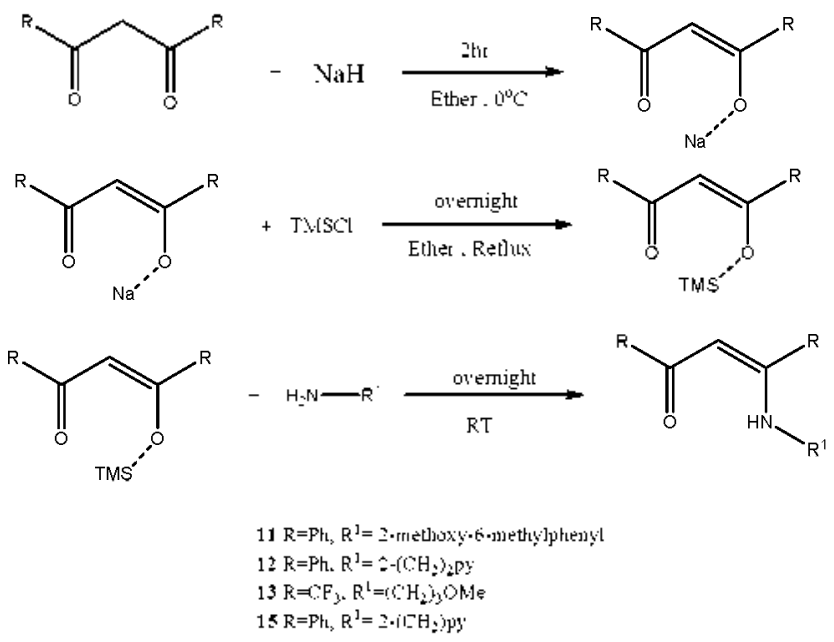

Scheme 2

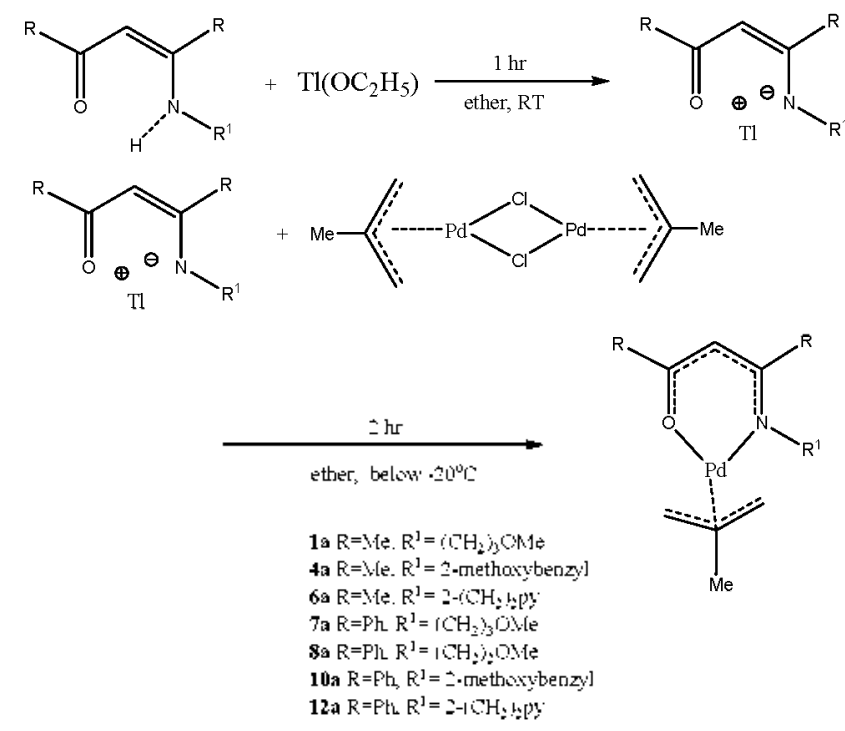

Scheme 3

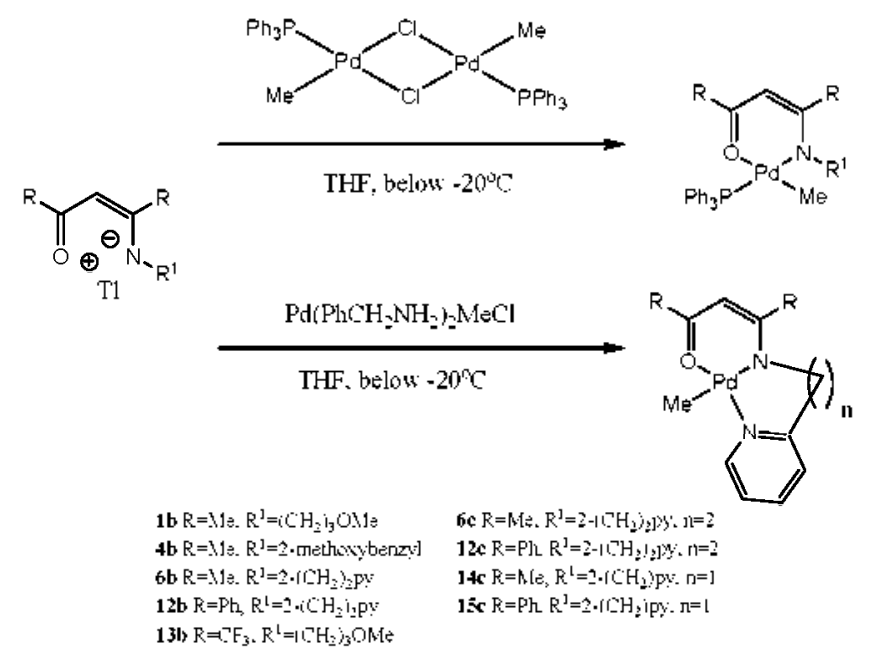

Scheme 4 have been obtained only in the class (III).

The class (I) of the complexes contain a $\eta^{3}$-methallyl ligand with functional $\beta$-ketoiminate one. In addition to $\beta$-ketoiminate ligands, both methyl and triphenylphosphine ligands and a methyl ligand are present in the class (II) and (III) of the complexes, respectively. The preparative methods are described in scheme 3 and 4 .

In the deprotonation step. $\mathrm{NaH}, \mathrm{Na}(\mathrm{OMe}) \mathrm{KH} . n-\mathrm{BuLi}$, and $\mathrm{Tl}(\mathrm{OEt})$ have been found to produce deprotonated $\beta$ ketoiminate anions successfully. However. starting palladium halides reacts with only thallium salts of $\beta$-ketoimine to produce corresponding target complexes with $\beta$-ketoiminate ligands.

In the preparation of methallyl complexes. 2-methyl allyl produced more stable complexes than nonsubstituted allyl. Even with 2-methyl allyl ligand. conplexes 13a is not stable enough to obtain satisfactory elemental analysis result. Considering the fact that corresponding phenyl $\beta$-ketoininates and 2-substituted allỵl analogues show much more stability. steric bulkiness seents to be more inportant for the stability than electronic effect in this class of complexes. As will be discussed below, the pendant bases in the functional $\beta$ ketoiminates fail to coordinate to the metal in the class (I) complexes.

The class (II) was originally designed to obtain alkyl complexes with tridentate $\beta$-ketoiminates but phosphines cannot be removed in the final products. The similar chemical shifts in ${ }^{31}$ P NMR spectra suggest that these complexes share almost identical coordination geometry. The palladium complex with the same formula as class (II) has been prepared in the reaction of $\mathrm{Pd}(\mathrm{COD}) \mathrm{MeCl}$ with potassium salt of a $\beta$-ketoiminate in the presence of $\mathrm{PPh}_{\text {s. }}{ }^{\text {dif }}$ It is rather surprising that ligands 14 and 15. where $\mathrm{N}$ bonded methylene chains are shortened by a methylene unit from the similar ligands 6 and 12, fail to produce complexes in the class (II). Since ligands 14 and 15 produced class (III) complexes successfully, steric hinderance exerted by bulky triphenylphosphine may be the reason.

In the preparation of class (III) of complexes. reactions between $\beta$-ketoiminates and $\mathrm{Pd}(\mathrm{COD}) \mathrm{MeCl}$, one of the most utilized starting complexes with no phosphines in palladium chemistry, fail to produce alkyl complexes with tridentate $\beta$ ketoiminates. In our efforts to find out the proper starting material, $\mathrm{Pd}\left(\mathrm{PhCH}_{2} \mathrm{NH}_{2}\right)=\mathrm{MeCl}$. obtained quantitatively from the reaction between $\mathrm{Pd}(\mathrm{COD}) \mathrm{MeCl}$ and $\mathrm{PhCH}_{2} \mathrm{NH}_{2}$, was found to produce target complex efficiently. Tridentate functional $\beta$-ketoiminates finally replace 2 anines and $\mathrm{Cl}$ ligands to produce complexes which can be formulated as ( $\mathrm{L}^{\wedge}$ $\left.\mathrm{O}^{\wedge} \mathrm{N}\right) \mathrm{PdR}$

Structuies of palladium complexes. The structures of three complexes. 1a $\mathbf{t b}$. and $\mathbf{1 2 c}$. one of which represents each class. respectively, were crystallographically determined and the crystallographic data were summarized in Table 1.

As shown in Figure 1, it is found that the methallyl coordinates to metal in a $\eta^{3}$ mode and the pendant base. an ethereal $\mathrm{O}$ atom. of the $\beta$-ketoinuinate is left uncoordinated in complex 1a The coordination geometry of complex $1 \mathrm{a}$ is best described as distorted square planar with $\mathrm{N}$ and $\mathrm{O}$ in the $\beta$ - 
ketoiminate ligand and 2 terminal carbon atoms of the $\pi$ methallyl ligand as the corners of the square. Interestingly a molecule adopts a structure whose geometrical parameters exhibit more contributing of (a) form, while the other more contributing of (b) form.

The pattern of bond distances from metal to methallyl ligand is different in two forms: in (a) terminal carbons (2.091(16) and 2.046(16) A) are located closer to metal than central carbon (2.192(14) $\AA$ ) but reverse (2.167(19) and $2.1+1(19) \AA$ vs $2.08(2) \AA$ ) is true in (b). Slight different Pd-terminal carbon distances may be due to different trans influences of $\mathrm{N}$ and $\mathrm{O}$ atom. In the (b) form. the difference in trans influence is less than that in the form (a). For $\left[\left(\eta^{3}-\right.\right.$ 2-methallyl)-Pd(trifluoroacetate) $]_{\text {I. }}$ where the terminal carbons of the methallyl ligands are trans to the oxygens of two trifluoroacetate bridging ligands, ${ }^{3+}$ and $\left[\mathrm{Pd}\left(\eta^{3}\right.\right.$-allyl $)($ tri-otolylphosphine)(triflate)]. where one of the terminal carbons is trans to the oxygen of triflate ligand, ${ }^{25}$ the $\mathrm{Pd}-\mathrm{C}$ distances $(2.096(5)$ and $2.073(5): 2.085(3) \AA$ ) are comparable to that found in (a) form. When the trans influence is quite different, the bond distances of Pd-allyl terminal carbon show substantial difference ${ }^{356}$ The allyl carbon-carbon distances C31-C32 (1.46(3) and 1.41(3) A) and C31-C33(1.41(3) and $1.36(3) \AA)$ are only slightly different. When the allyl coor- dinates in a $\eta^{3}, \eta^{2}$ mode as found in $\left.\left[\mathrm{Ni}\left(\mathrm{P}^{\wedge} \mathrm{O}\right)(\text { ally } 1)\right]^{+}\right]^{\text {? }}$ ? substantial differences in allyl carbon-carbon distances (1.373(22). 1.314(24) A) and metal-carbon distances $\left(\mathrm{M}-\mathrm{C}_{\mathrm{t}}\right.$, $1.995(11)$ and $\left.2.024(11) ; \mathrm{M}-\mathrm{C}_{c} .1 .998(11) \AA\right)$ are observed. In complex 1a. two carbon-carbon bond distances in an methallyl ligand are slightly different: 1.46(3). 1.41(3) and $1.41(3), 1.36(3) \AA$. respectively. In a free $\beta$-ketoimine ligand. enamine form (b) is predominant but it is interesting that two forms, enamine $\left(\mathrm{C} 5^{\circ}-\mathrm{C} 6^{\circ}, 1.31(2): \mathrm{C}^{\circ}-\mathrm{C} 7^{\prime}, 1.42(2): \mathrm{C}^{\circ}-\mathrm{O} 1^{\prime}\right.$, $\left.1.270(17): \mathrm{C}^{\circ}-\mathrm{NI}^{\circ}, 1.33(2): \mathrm{C}^{\circ}-\mathrm{NI}^{\circ} .1 .44(2) \mathrm{A}\right)$ and enolimine (C5-C6, 1.560(18); C6-C7. 1.37(2); C7-O1. 1.37(2); C5-NI. 1.25(2): C4-NI, 1.488(17) A), are present simultaneously in a complex. There is no indication of any inter-

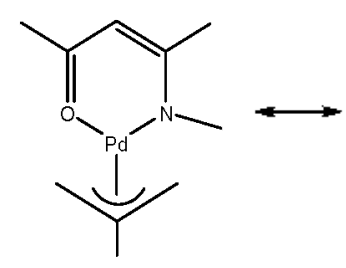

(a)

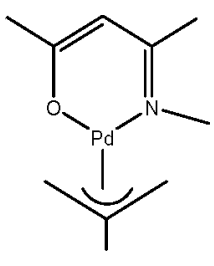

(b)
Scheme 5

Table 1. Crystallographic Data for Compounds 1a and 12c.

\begin{tabular}{|c|c|c|c|}
\hline & la & $12 \mathrm{c}$ & $4 \mathrm{~b}$ \\
\hline Empirical formula & $\mathrm{C}_{4} \mathrm{H}_{2} \mathrm{~N}_{2} \mathrm{O}_{4} \mathrm{Pd}_{2}$ & $\mathrm{C}_{23} \mathrm{H}_{23} \mathrm{~N}_{2} \mathrm{OPd}$ & $\mathrm{C}_{32} 5_{0} \mathrm{H}_{35} \mathrm{ClNO} \mathrm{PPd}_{2}$ \\
\hline Formula weight & 871.74 & 448.83 & 644.44 \\
\hline Temperature & $293(2) \mathrm{K}$ & $173(2) \mathrm{K}$ & $298(2) \mathrm{K}$ \\
\hline Wavelength & $0.71073 \AA$ & $0.71073 \AA$ & $0.71073 \AA$ \\
\hline Space group & triclinic, $\mathrm{PI}$ & Orthorhombic, P2(1)2(1)2(1) & Orthorhombic, $\mathrm{Pbcn}$ \\
\hline a & $10.187(8) \AA$ & $10.0740(8) \AA$ & $31.334(3) \AA$ \\
\hline $\mathrm{b}$ & $10.656(8) \AA$ & $10.9425(8) \AA$ & $12.0633(13) \AA$ \\
\hline c & $10.792(9) \AA$ & $18.1835(13) \AA$ & $16.0742(17) \AA$ \\
\hline$\alpha$ & $91.505(14)^{\circ}$ & $90^{\circ}$ & $90^{\circ}$ \\
\hline$\beta$ & $100.561(14)^{\circ}$ & $90^{\circ}$ & $90^{\circ}$ \\
\hline$\gamma$ & $114.820(13)^{\circ}$ & $90^{\circ}$ & $90^{\circ}$ \\
\hline Volume & $1038.2(14) \AA^{3}$ & $2004.5(3) \AA^{3}$ & $6075.9(11) \AA^{3}$ \\
\hline 2 & 2 & 4 & 8 \\
\hline Density (calc.) & $2.789 \mathrm{Mg} / \mathrm{m}^{3}$ & $1.487 \mathrm{Mg} / \mathrm{m}^{3}$ & $1.409 \mathrm{Mg} / \mathrm{m}^{3}$ \\
\hline Absorption coeft. & $1.813 \mathrm{mmm}^{-1}$ & $0.940 \mathrm{~mm}^{-1}$ & $0.781 \mathrm{~mm}^{-1}$ \\
\hline$F(000)$ & 904 & 912 & 2648 \\
\hline Crystal size & $0.38 \times 0.30 \times 0.18 \mathrm{~mm}^{3}$ & $0.18 \times 0.20 \times 0.35 \mathrm{~mm}^{3}$ & $0.20 \times 0.25 \times 0.50 \mathrm{~mm}^{3}$ \\
\hline Reflections collected & 5496 & 12831 & 36782 \\
\hline Independent reflections & $4622[\mathrm{R}(\mathrm{int})=0.0329]$ & $4735[\mathrm{R}(\mathrm{int})=0.0536]$ & $7355[\mathrm{R}($ int $)=0.0881]$ \\
\hline Data / restraints / parameters & $4622 / 3 / 487$ & $4735 / 0 / 245$ & $7355 / 0 / 349$ \\
\hline Goodness-of-fit on F2 & 0.996 & 0.973 & 1.124 \\
\hline Final $R$ indices $[I>2 \sigma(I)]$ & $\mathrm{R}_{1}=0.0426, \mathrm{wR}_{2}=0.1057$ & $\mathrm{R}_{1}=0.0338, \mathrm{wR}=0.0569$ & $\mathrm{R}_{1}=0.0797, \mathrm{wR}_{2}=0.1746$ \\
\hline R indices (all data) & $\mathrm{R}_{1}=0.0515, \mathrm{wR}_{2}=0.1102$ & $\mathrm{R}_{1}=0.0457, \mathrm{wR}_{2}=0.0596$ & $\mathrm{R}_{1}=0.1368, \mathrm{wR}_{2}=0.2013$ \\
\hline Absolute structure parameters & $0.14(7)$ & $-0.02(3)$ & none \\
\hline Largest diff. peak and hole & 0.816 and $-0.929 \mathrm{e} . \AA^{-3}$ & 0.944 and $-0.549 \mathrm{e} A^{-3}$ & 1.063 and -2.160 e. $A^{-3}$ \\
\hline
\end{tabular}


(a)
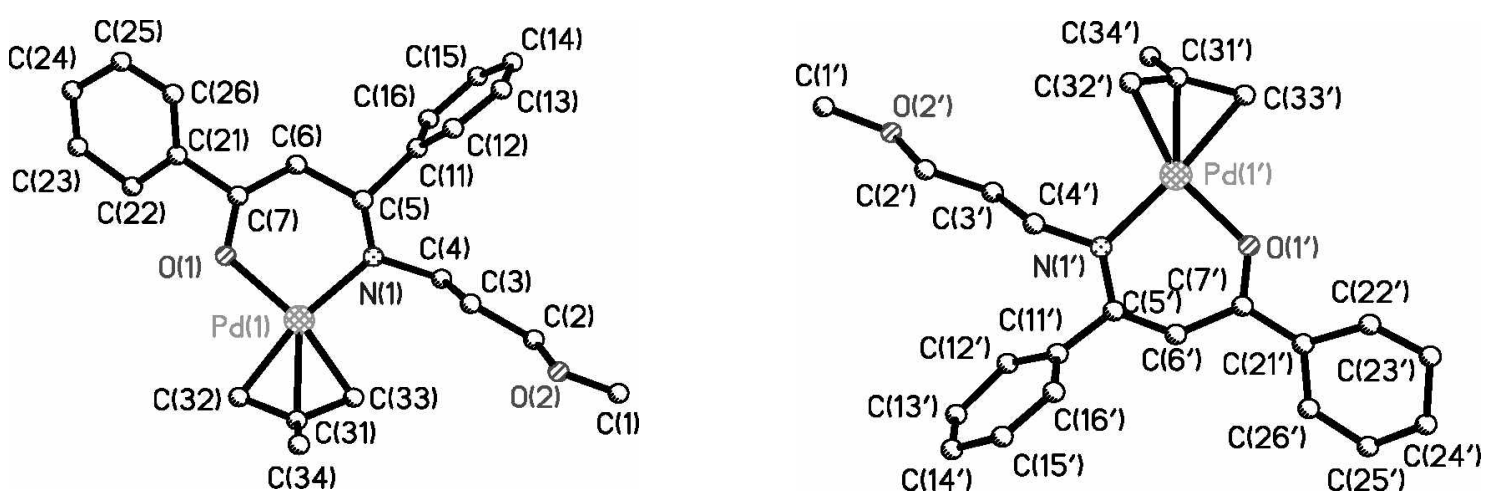

(b)

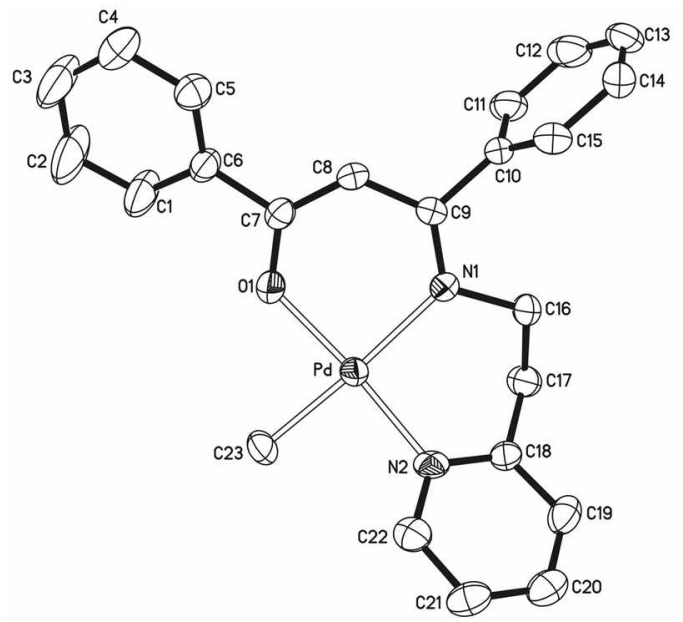

(c)

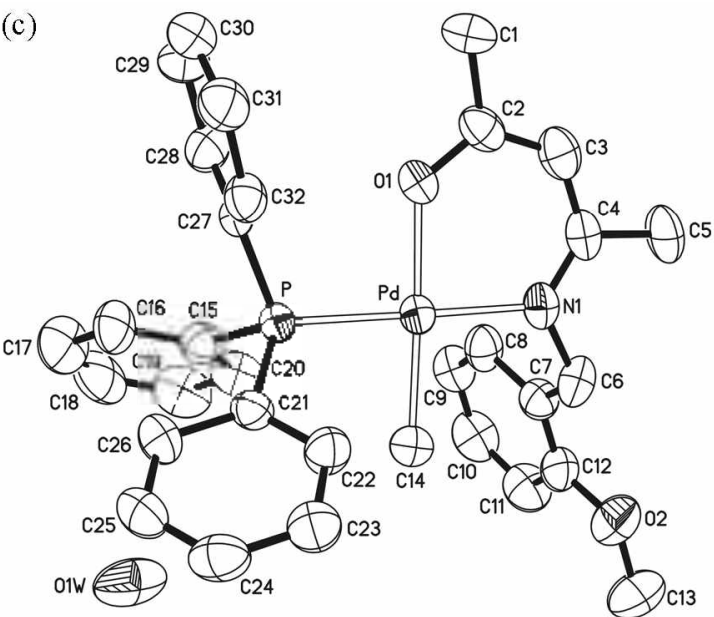

Figure 1. (a) The structure of 1a. Selected bond distances and angles: $\mathrm{Pd}(1) \mathrm{j}-\mathrm{N}(1) 2.044(16), \mathrm{Pd}(\mathrm{l}) \mathrm{C}(33) 2.046(18), \mathrm{Pd}(1) \mathrm{O}(1) 2.051(14)$,

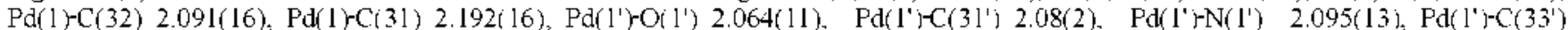
2.141(19), Pd( $\left.1^{\prime}\right) C\left(32^{\circ}\right) 2.167(19), O\left(1^{\prime}\right)-C\left(7^{\circ}\right) 1.270(17), O(1) C(7) 1.32(2), N(1)-C(5) 1.25(2), \mathrm{N}(1)-C(4) 1.488(17), N\left(1^{\circ}\right)-C\left(5^{\prime}\right) 1.33(2)$,

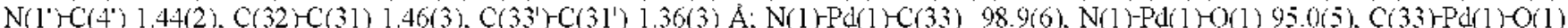
163.0(6), N(1)-Pd(1)-C(32) 166.9(7), C(33)-Pd(1)-C(32) 69.1(8), O(1)-Pd(1)-C(32) 96.1(7), N(1)-Pd(1)-C(31) 132.0(6), C(33)-Pd(1)-C(31)

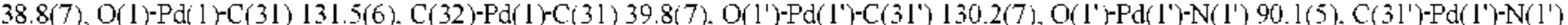

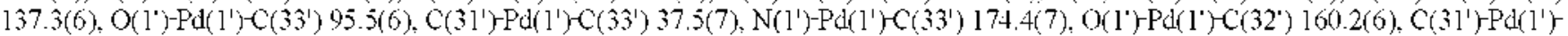
$\mathrm{C}\left(32^{\prime}\right) 38.8(8), \mathrm{N}\left(1^{\prime}\right) \mathrm{Pd}\left(\mathrm{l}^{\prime}\right) \mathrm{C}\left(32^{\prime}\right) 106.5(7), \mathrm{C}\left(33^{\prime}\right) \mathrm{Pd}\left(\mathrm{l}^{\circ}\right) \mathrm{C}\left(32^{\prime}\right) 68.0(8)^{\circ}$ (b) The structure of $12 \mathrm{c}$. Selected bond distances and angles: $\mathrm{Pd}-$ $\mathrm{O}(1) 2.016(2), \mathrm{Pd}-\mathrm{C}(23) 2.037(3), \mathrm{Pd}-\mathrm{N}(2) 2.040(3) \mathrm{Pd}-\mathrm{N}(1) 2.100(3), \mathrm{O}(1) \mathrm{C}(7) 1.298(4), \mathrm{N}(1) \mathrm{C}(9) 1.303(4), \mathrm{N}(1) \mathrm{C}(16) 1.472(4), \mathrm{N}(2)$ $\mathrm{C}(18) 1.341(4), \mathrm{N}(2)-\mathrm{C}(22) 1.354(4), \mathrm{C}(7)-\mathrm{C}(8) 1.373(4), \mathrm{C}(8)-\mathrm{C}(9) 1.437(4) \mathrm{A} ; \mathrm{O}(1) \mathrm{Pd}-\mathrm{C}(23) 84.70(13), \mathrm{O}(1)-\mathrm{Pd}-\mathrm{N}(2) 174.13(10), \mathrm{C}(23)-$ $\mathrm{Pd}-\mathrm{N}(2) 91.43(13), \mathrm{O}(1)-\mathrm{Pd}-\mathrm{N}(1) 91.30(10), \mathrm{C}(23)-\mathrm{Pd}-\mathrm{N}(1) 175.55(14), \mathrm{N}(2) \mathrm{Pd}-\mathrm{N}(1) 92.71(10)^{\circ}$. (c) The structure of $4 \mathrm{~b}$. Selected bond

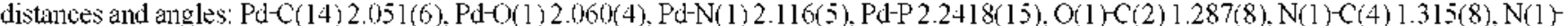
$\mathrm{C}(6) 1.458(8), \mathrm{C}(2) \mathrm{C}(3) 1.387(10), \mathrm{C}(3)-\mathrm{C}(4) 1.402(9) \AA: \mathrm{C}(14)-\mathrm{Pd}-\mathrm{O}(1) 175.5(3), \mathrm{C}(14)-\mathrm{Pd}-\mathrm{N}(1) 92.6(2), \mathrm{O}(1)-\mathrm{Pd}-\mathrm{N}(1) 90.92(19), \mathrm{C}(14)$ -Pd-P 87.5(2), O(1)-Pd-P 89.07(13), N(1)-Pd-P 177.17(14) ${ }^{\circ}$.

action between metal and the ethereal $\mathrm{O}$ atom.

In the crystal stnicture of complex $4 \mathbf{b}$ as reported elsewhere, ${ }^{23}$ the pendant base, an ethereal $O$ atom, of the $\beta$ ketoiminate is left uncoordinated as in complex 1a. The structure of complex $\mathbf{t b}$ shows a slightly distorted square planar arranyement comprising the plane with $\mathrm{N}$ and $\mathrm{O}$ in the $\beta$-ketoiminate ligand. a phosphine, and a methyl ligand. As expected from the small coupling constant $(3.0-3.6 \mathrm{~Hz})$ between phosphine and methyl protons and chelating nature of $\beta$-ketoiminate. the methyl group on palladium is positioned cis to the phosphine. The Pd-P bond length of $2.2418(15) A$ is comparable to those in phosphine complexes (2.20-2.32 $\AA){ }^{\geq, 2,3,3(1}$ The Pd-methyl bond distance $(2.05 \mathrm{I}(6) \AA)$ is rather long but in agreement with values (2.01-2.05 $\AA$ ) for related complexes. ${ }^{z 8-34}$ The $\beta$-ketoiminate ligand can be described as an enolimine. similar to salicylaldiminate. on the basis of bond lengths (C2-O. 1.287(8): C2-C3.1.387(10): C3-C4. 1.402(9): C4-N1, $1.315(8) \mathrm{A}$ )

In the crystal structure of complex $12 \mathrm{c}$. the internal base, an $\mathrm{N}$ atom in the pyridine ring. of the $\beta$-ketoiminate finally coordinates to the metal. The structure is slightly distorted square planar and the functional $\beta$-ketoiminate ligand spans almost in the same plane around the metal. The Pd-nethyl bond distance $(2.037(3) \mathrm{A})$ is in agreement with values $(2.01-2.05 \AA)$ for related complexes ${ }^{28-34}$ but shorter than that in $\mathbf{t b}$ possibly due to lack of the phosphine. The $\beta$-ketoiminate ligand can be also described as an enolimine. similar to salicylaldiminate. on the basis of bond lengths (C7-Ol, 1.298 (4): C7-C8. 1.373(4): C8-C9, 1.437(4): C9-N1. 1.303(8): $\mathrm{NI}-\mathrm{Cl} 6,1.472(4) \mathrm{A}$ ). The Pd-N bond distance of $\beta$-ketoiminate ligand $(2.100(3) \AA)$ is longer than that of pyridine $(2.040(3)$ A) possibly due to partial contribution of enamine character. 
Table 2. Norbonene Polymerization Results without cocatalysts.

\begin{tabular}{cccc}
\hline Catalyst & Solvent & Yield (\%) & $\begin{array}{c}\text { Activity } \\
\text { (kg/Pdmolih) }\end{array}$ \\
\hline 1b & & 20.0 & 14.3 \\
6b & & 13.0 & 9.0 \\
$\mathbf{1 2 b}$ & $\mathrm{CH}_{2} \mathrm{Cl}_{2}$ & 15.0 & 11.5 \\
$12 \mathbf{c}$ & & 16.5 & 9.1 \\
$14 \mathbf{c}$ & & 23.0 & 8.9 \\
$15 \mathbf{c}$ & & 17.5 & 8.5 \\
\hline
\end{tabular}

Amount of catalyst: $0.01 \mathrm{~g}$. [Monomer] [catalyst] $=1000$. solvent volume $=3 \mathrm{~mL}$, reaction time $=6 \mathrm{~h}$. reaction temperature $=25^{\circ} \mathrm{C}$.

Table 3. Norbonene Polymerization Results with a Cocatalyst, $\left[\mathrm{H}\left(\mathrm{Et}_{2} \mathrm{O}\right)_{2}\right]^{+} \mathrm{BAT}_{4}^{1^{-}}$.

\begin{tabular}{|c|c|c|c|}
\hline Catalyst & Solvent & Yield (\%) & $\begin{array}{c}\text { Activity } \\
\text { (kg/Pdmol/hr) }\end{array}$ \\
\hline \multirow[t]{3}{*}{$\mathrm{Pd}(\text { methallyl) } \mathrm{Cl}]_{2}$} & $\mathrm{CH}_{2} \mathrm{Cl}_{2}$ & 95 & 263 \\
\hline & $\mathrm{PhCl}$ & 77 & 204 \\
\hline & $\mathrm{PlhMe}$ & 13.5 & 21.3 \\
\hline \multirow[t]{3}{*}{$6 a$} & $\mathrm{CH}_{2} \mathrm{Cl}_{2}$ & 35 & 191 \\
\hline & $\mathrm{PhCl}$ & 60 & 328 \\
\hline & $\mathrm{PhMe}$ & 25 & 138 \\
\hline \multirow[t]{3}{*}{$1 b$} & $\mathrm{CH}_{2} \mathrm{Cl}_{2}$ & 43.5 & 324 \\
\hline & $\mathrm{PhCl}$ & 52.3 & 390 \\
\hline & $\mathrm{PlhMe}$ & 21.1 & 157 \\
\hline $6 \mathrm{~b}$ & $\mathrm{CH}_{2} \mathrm{Cl}_{2}$ & 45.2 & 295 \\
\hline \multirow[t]{3}{*}{$12 b$} & $\mathrm{CH}_{2} \mathrm{Cl}_{2}$ & 57.5 & 456 \\
\hline & $\mathrm{PhCl}$ & 94.6 & 749 \\
\hline & $\mathrm{PhMe}$ & 14.1 & 112 \\
\hline \multirow[t]{3}{*}{$13 b$} & $\mathrm{CH}_{2} \mathrm{Cl}_{2}$ & 85.1 & 771 \\
\hline & $\mathrm{PhCl}$ & 90.2 & 810 \\
\hline & $\mathrm{PhMe}$ & 56.6 & 509 \\
\hline $6 c$ & $\mathrm{CH}_{2} \mathrm{Cl}_{2}$ & 84.5 & 477 \\
\hline $6 c^{\prime \prime}$ & $\mathrm{CH}_{2} \mathrm{Cl}_{2}$ & 62.7 & 355 \\
\hline $6 c^{b}$ & $\mathrm{CH}_{2} \mathrm{Cl}_{2}$ & 16.5 & 93 \\
\hline \multirow[t]{3}{*}{$12 c$} & $\mathrm{CH}_{2} \mathrm{Cl}_{2}$ & 67.2 & 531 \\
\hline & $\mathrm{PhCl}$ & 48.8 & 379 \\
\hline & $\mathrm{PhMe}$ & 40.7 & 318 \\
\hline $14 c$ & $\mathrm{CH}_{2} \mathrm{Cl}_{2}$ & 79.4 & 1026 \\
\hline $15 \mathrm{c}$ & $\mathrm{CH}_{2} \mathrm{Cl}_{2}$ & 86.6 & 657 \\
\hline \multirow[t]{3}{*}{16} & $\mathrm{CH}_{2} \mathrm{Cl}_{2}$ & 90.7 & 329 \\
\hline & PhCl & 97.3 & 348 \\
\hline & PliMe & 62.9 & 224 \\
\hline
\end{tabular}

Reaction temperature: $25^{\circ} \mathrm{C}$. solvent volume $=15 \mathrm{~mL}$. [monomer] [catalyst $]=1000$, reaction time $=5 \mathrm{~min} .14 \mathrm{c}=3 \mathrm{~min} .{ }^{a}[$ cocatalyst $]:$ [catalyst $]=4 . "$ cocatalyst $]$ [catalyst $]=10$

However. the Pd-N bond distance of pyridine is rather shorter than those found in phosphino-pyridine complex $(2.21-2.23$ A) ${ }^{3(1}$ This may be ascribed to the presence of bulky substituents on the py ridine ring.

Similar enolimine structures of coordinated $\beta$-ketoiminate ligands $\left(\mathrm{C}_{1}-\mathrm{O}-\mathrm{O} .285(2), 1.2893(19): \mathrm{C} \cdot \mathrm{-N} .1 .320(2), 1.323(2)\right.$; $\left.\mathrm{C}_{0}-\mathrm{C}_{6}, 1.362(3), 1.368(2): \mathrm{C}_{\mathrm{N}}-\mathrm{C}_{1}, 1.411(2), 1.401(2)\right)\left(\mathrm{C}_{\mathrm{O}}\right.$ $\mathrm{C}_{\mathrm{N}}$ and $\mathrm{C}_{C}$ are carbon next to $\mathrm{O}$. one next to $\mathrm{N}$. and centeral one of the $\beta$-ketoiminate ligand. respectively) were reported in the $\mathrm{Ni}(\beta \text {-ketoiminate ligand })_{2}$ complexes. ${ }^{\text {Ifiel }}$

Polymerization of norbomene. During our efforts to investigate the reactivities of the class (I) complexes containing $\beta$ ketoiminate ligand, the inactivity of these complexes towards
Table 4. Norbomene Polymerization Results with a Cocatalyst, MMAO

\begin{tabular}{|c|c|c|c|}
\hline Catályst & Solvent & Yield (\%) & $\begin{array}{c}\text { Activity } \\
\text { (hg/Pdmol.hr) }\end{array}$ \\
\hline $1 \mathrm{~b}$ & PhMe & 62.0 & 7600 \\
\hline $6 \mathrm{~b}$ & $\mathrm{CH}_{2} \mathrm{Cl}_{2}$ & 95.0 & 24600 \\
\hline \multirow[t]{3}{*}{$12 \mathrm{~b}$} & $\mathrm{CH}_{2} \mathrm{Cl}_{2}$ & 97.5 & 26100 \\
\hline & $\mathrm{PhCl}$ & 95.0 & 22000 \\
\hline & $\mathrm{Pl} \mathrm{Me}$ & 41.5 & 5030 \\
\hline \multirow[t]{3}{*}{$13 \mathrm{~b}$} & $\mathrm{CH}_{2} \mathrm{Cl}_{2}$ & 91.5 & 29300 \\
\hline & $\mathrm{PhCl}$ & 89.0 & 24200 \\
\hline & PhMe & 73.5 & 11200 \\
\hline $6 c$ & $\mathrm{CH}_{2} \mathrm{Cl}_{2}$ & 94.0 & 37100 \\
\hline \multirow[t]{3}{*}{$12 \mathrm{c}$} & $\mathrm{CH}_{2} \mathrm{Cl}_{2}$ & 91.0 & 51400 \\
\hline & PhCl & 94.0 & 53000 \\
\hline & PhMe & 65.5 & 34800 \\
\hline $14 c$ & $\mathrm{CH}_{2} \mathrm{Cl}_{2}$ & 97.5 & 93400 \\
\hline $15 c$ & $\mathrm{CH}_{2} \mathrm{Cl}_{2}$ & 95.0 & 77100 \\
\hline \multirow[t]{3}{*}{16} & $\mathrm{CH}_{2} \mathrm{Cl}_{2}$ & 100 & 28300 \\
\hline & $\mathrm{PhCl}$ & 92.5 & 20500 \\
\hline & PhMe & 48.0 & 6200 \\
\hline
\end{tabular}

olefin, acetylene, and phosplines is rather suprising. In NMR experiments. no changes are monitored after addition of external $L$ type ligands even after a day. This suggests that $\beta$ ketoiminate and methallyl ligands in these complexes coordinate rather strongly and no transformation of bonding modes $\left(\eta^{3} \rightarrow \eta^{1}\right)$ of the methallyl ligand occurs under these conditions. Finally. removal of the methallyl ligand by introduction of proton source. HBAr's was tried to activate for olefin poly merization and a success was observed. The polymerization results of the selected conplexes are sunmarized in Table 3.

Without cocatalysts. class (I) complexes are found to be inactive but class (II) and (III) conplexes show activities even though much slower reaction rates and lower activities than those with cocatalysts are observed in the olefin polymerization (Table 2). The polymers are insoluble in common organic solvents. indicating high molecular weight or highly crosslinked ones. As shown in Table 3. the higher activities in the class (I) complexes on activation with cocatalysts are generally observed in polar solvents $\left(\mathrm{PhCl}>\mathrm{CH}_{2} \mathrm{Cl}_{2}>\mathrm{PhMe}\right.$ ), indicating the polar character of the active species. while reference complex. $[\mathrm{Pd}(\text { methally }) \mathrm{Cl}]_{2}$. showed the highest activity in $\mathrm{CH}_{2} \mathrm{Cl}_{2}$.

In the class (II) complexes. activation can be done by $\mathrm{HBAr}^{\prime}+$ or MAO. It is found that MAO is more efficient than $\mathrm{HBAr}^{\prime}$, by at least lor 2 order in activity as shown in Table 3 and 4 . In this class, activity increases in the following order; $\mathbf{1 b}<\mathbf{6} \mathbf{b}<\mathbf{1 2 b}<\mathbf{1 3 b}$ (HBAr', activation). Electron-withdrawing nature of $\mathrm{Ph}_{\text {and }} \mathrm{CF}_{3}$ may render the metal center to be more electrophilic to facilitate the coordination of olefin and higher activity:

All the activities obtained in this study are less than but in the same order as one with a nonfunctionalized $\beta$-ketoiminate reported earlier. ${ }^{1+1[1}$ However, much more catalyst and MAO were used in that case $([\mathrm{NB}] /[\mathrm{Pd}]=200$. $[\mathrm{Al}] /[\mathrm{Pd}]=5000)$. 
On activation with $\mathrm{HBAr}^{\prime}{ }_{4}$. the solvent effect is almost same as that in the $\mathrm{L}^{* 1}$ class complexes: $\mathrm{PlCCl}>\mathrm{CH}_{2} \mathrm{Cl}_{2}>\mathrm{PhMc}$. suggesting the similar polar nature of active species. On activation of MMAO. the same solvent effect. higher activity. in polar solvents. was observed even though the activities in methylene chloride are slightly higher than those in chlorobenrenc. Howcrer, it is not as significant as that found with HBAr'.. Complex 16. $\mathrm{Pd}\left(\mathrm{PhCH}_{2} \mathrm{NH}_{2}\right)_{2} \mathrm{MeCl}$. showed the similar solvent effect on aclivity.

In the class (III). activity increases in the following order: $6 \mathrm{c}<12 \mathrm{c}<15 \mathrm{c}<14 \mathrm{c}$ (HBAr., or MMAO activation). In this class. it is crident that activity in toluene is in the same order as those in polar solvents even though it is still lower. The reason for this plenomenon is not clear yet. Addition of more than + equivalents of $\mathrm{HBAr}^{\circ}$ resulted in much less activity. suggesting decomposition of active species to inaclive onc. The length of backbone also affects the activ ity possibly duc to different rate of opening a vacant site for olefin. As cxpected from the ring sirain. 15c with a short backbone showed higher activity than $\mathbf{1 2 c}$. It is interesting to observe reverse effect of the substituent on the activity in a higher ring-strain system: 15c with electron-withdraw ing $\mathrm{Ph}$ substituents showed less activity than $14 \mathrm{c}$ with Me.

As expecied from the easy formation of a vacant site for the olefin coordination. the polymerization activity on activation of HBAr'. generally increases with the following order: $[\mathrm{Pd}($ methallyl $)(\beta$-ketoiminate $)]<\mid \mathrm{Pd}(\mathrm{Mc})\left(\mathrm{PPh}_{3}\right)(\beta$-ketoiminate $) \mid$ $<\mid \mathrm{Pd}(\mathrm{Mc})(\beta$-ketoiminatc) $\mid$ under the same conditions. On MMAO activation. 14c showed the best activity in this study.

Polymerization mechanism. As mentioned above. the class (I) of the complexes withoul cocatalysts was inactive and should be activated by the addition of HBAr', for the olefin polymerization. There are two possible pallways described in Sclieme 6 in the reaction with HBAr's. In order to determine the actual outcome. an NMR experiment has been done.

Initially it is thought that proton removes the metlallyl ligand preferentially but contran to our expectation. a frec $\beta$ keloimine with typical peak at $10.8 \mathrm{ppm}$ was observed in an NMR experiment. suggesting the preferential protolysis of $\beta$ keloimine occurs.

However. $\mid \mathrm{Pd}($ methally])Cl| , which should produce the same intermediate on activation with NaBAr'. showed less aclivity towards norbornene polymerization and different activities with complexes containing olher $\beta$-ketoiminate ligands were observed even though the differences are not

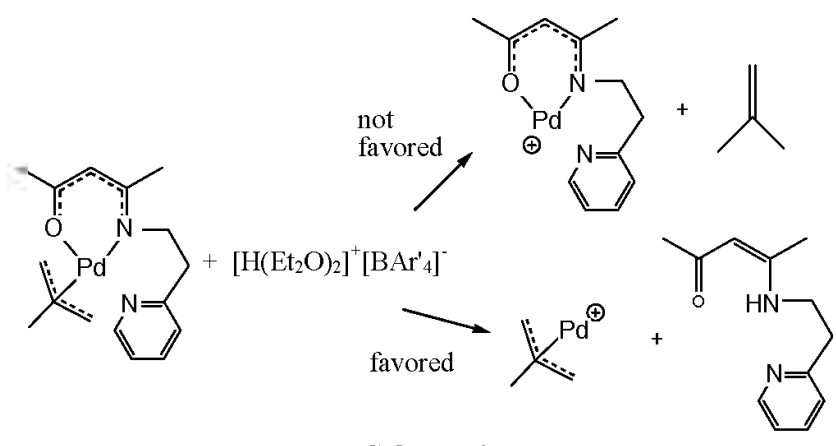

Scheme 6

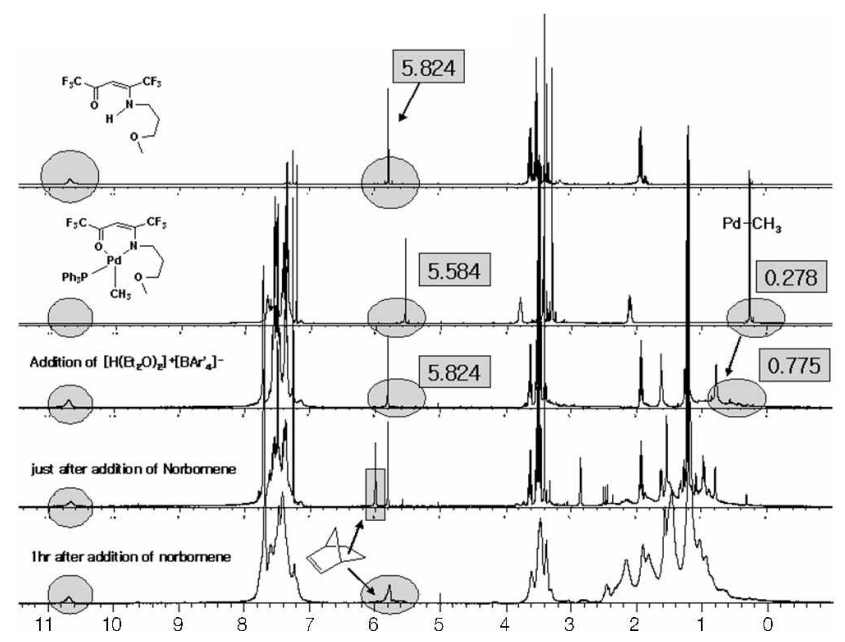

Figure 2. 'I NMR spectra of the reactions between 13b and [H( $\left.\left.\mathrm{Hit}_{2} \mathrm{O}_{2}\right]^{\prime}\right] \mathrm{B} / \mathrm{Ar}_{4}{ }^{\prime}$

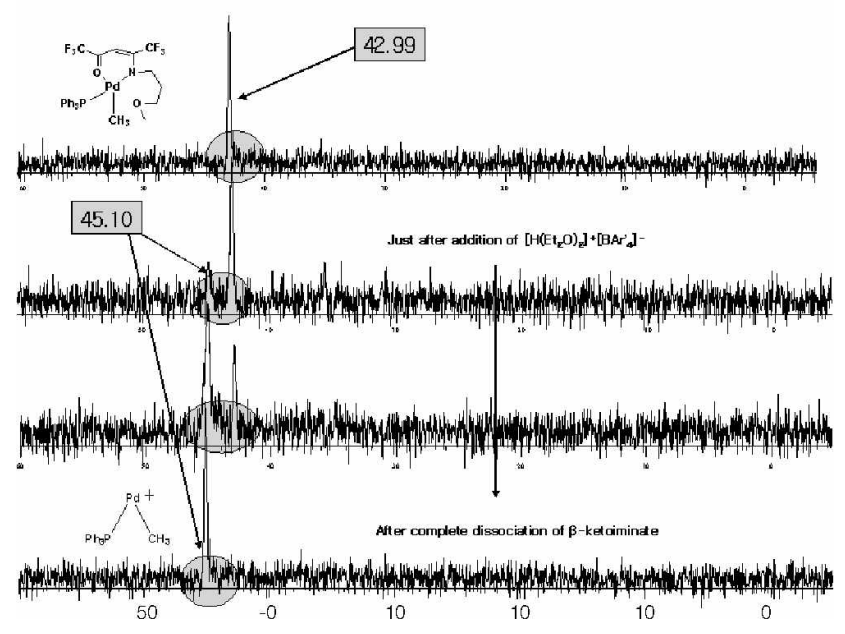

Figure 3. "P NMR spectra of the reactions between $13 \mathrm{~b}$ and $\left[\mathrm{H}\left[\left(1, \mathrm{~L}_{2} \mathrm{O}\right)_{2}\right]^{\prime} 1 \mathrm{~B} / \mathrm{Ar}_{1}\right.$

great. The different and generally higher polymerization activities than $[\mathrm{Pd}(\text { methallyl }) \mathrm{Cl}]_{2}$ in this class of complexes also found. Preactivation of catalysts by introducing $\mathrm{HBAr}^{\prime}$. before addition of norbornene resulted in no polymerization and only activation of the precatalysts in the presence of olefins leads to poly merization. suggesting the quite unstable character of active species

Unfortunately. the polymers are not soluble in common organic solvents and the characteristics of the poly mer cannot be further investigated.

In the class (II) of the complexes. detailed mechanism was investigated with $\mathbf{1 3 b}$. In this case. free $\beta$-ketoimine ligand was formed while mellyyl and phosphine ligands were survived on activation with HBAr'., The survival of methyl and phosphine ligands was confinned by ${ }^{1} \mathrm{H}$ and ${ }^{\$ 1} \mathrm{P}$ NMR spectra (Figure 2 and 3). The chemical shift of melyyl ligand was downshified from 0.278100 .775 . which is often observed during the process of obtaining + charge in the complex.

The chemical shift of $\mathrm{PPl}_{3}$ was almost same $(+2.99 \mathrm{ppm}$ w. 45. I0 $\mathrm{ppm}$ ) but quite different from those of free $\mathrm{PPh}_{3}(0$ ppm). Possible formation of phosphonium salt can be excluded 


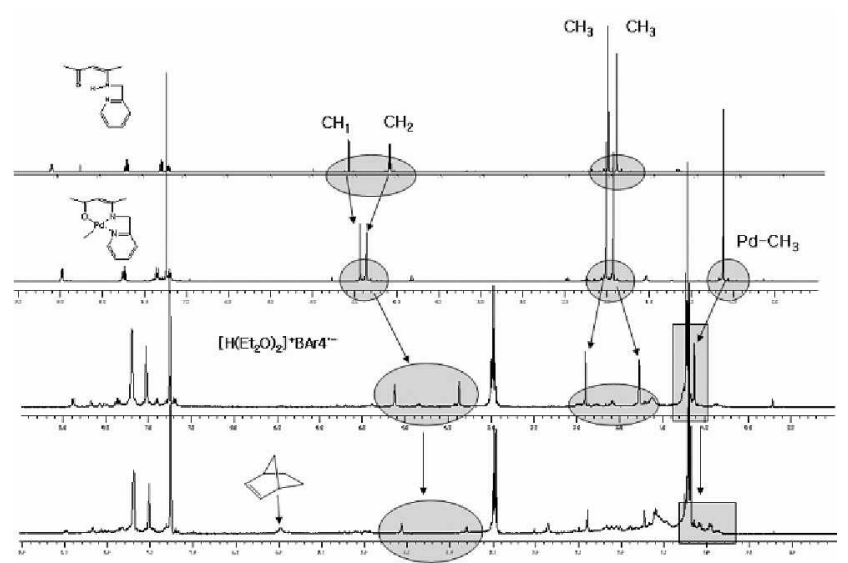

Figure 4. ${ }^{1} \mathrm{H}$ NMR spectra of the reactions between $\mathbf{1 4 c}$ and $\left[\mathrm{H}\left(\mathrm{Et}_{2} \mathrm{O}\right)_{2}\right]^{+} \mathrm{BAr}_{1}{ }^{1-}$

excluded by the chemical shift obtained from the reaction between $\mathrm{PPh}_{3}$ and $\mathrm{HBAr}_{4}$ (14.932 and 11.903 ppm). $\mathrm{HBF}_{\downarrow}$ (12.506 and $9.293 \mathrm{ppm}$ ) and $\mathrm{HCl}$ (3.243 ppm). Unfortunately, the splitting of the methyl peak in the product by the coordinated phosphine was not observed but a little broader peak $\left(\mathrm{W}_{1: z}=12 \mathrm{~Hz}\right.$ ) was resulted. Therefore, it is assumed that $\left[\mathrm{Pd}(\mathrm{Me})\left(\mathrm{PPh}_{3}\right)\right]^{-}$was formed as an active species. The activity difference between $\mathbf{1 3 b}$ and $\mathbf{1 b}$ seems to rely on the tendency of protoly sis of $\beta$-ketoiminate. Since ligand 13 is more acidic than 1 . it is expected that the deprotonated $\mathbf{1 3}$ is less basic and $\mathrm{M}-\mathrm{L}$ bond strength is weaker.

In the class (III) of the complexes. preferential removal of methyl ligand is assumed but it is survived after addition of approximate 1 equivalent of $\mathrm{HBAr}_{4}$ (Figure 4). However. the methyl peak appeared downfield (from $0.6+$ to 1.12 ) significantly in the $\mathrm{H}$ NMR spectrum. Formation of a free $\beta$ ketoiminate ligand was not observed. The peaks representing $\mathrm{CH}$ of $\beta$-ketoiminate and $\mathrm{CH}_{2}$ next to the pyridine shift from 4.95 and 4.89 to 3.87 and 4.60 , respectively. while integration ratio changes from $1: 2$ to $2: 2$. Two methyl peaks of $\beta$ ketoiminate also shift from 2.04 and 1.95 to 2.40 and 1.76 . In the free $\beta$-diketone ligands, methine peaks in the keto form appear in the range of 3.5-4.0 ppm. However. this complex was found to convert to pyridinium complexes during the accumulation of the ${ }^{13} \mathrm{C}$ NMR data overnight. Therefore. confirmation of $\mathrm{CH}_{2}$ (resonance at around $70 \mathrm{ppm}$ ) cannot be done but one peak at 65.864 ppm was observed. From these observations. it may be concluded that protonation would lead to formation of the intermediate where $\beta$-ketoimine coordinates to the metal in the keto form (Scheme 7).

$1+c$ with electron-donating substituent Me showed higher activity than $15 \mathrm{c}$ with $\mathrm{Ph}$ possibly due to stabilization of protonated intermediate. This protonated $\beta$-ketoiminate would deviate from the planity due to change of hybridization, which leads to dissociation of internal base. Also complexes with longer backbone showed lower activity due to lower tendency of dissociation of internal base which is originated from ligher tolerance toward distortion with lower ring strain of 6-member ring.

Even though reported examples of this type of coordination in the $\beta$-ketoiminate complexes are rare, there are some exam-

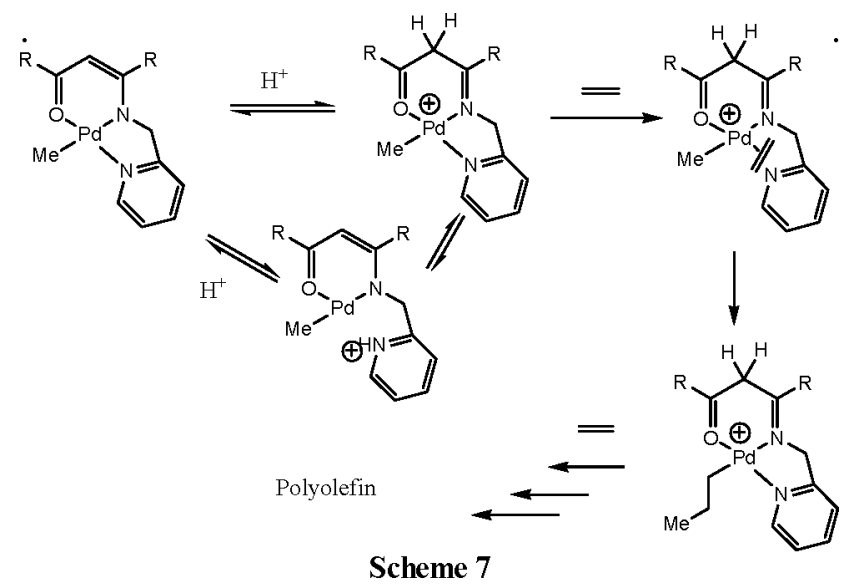

ples in the $\beta$-diketonate complexes. ${ }^{26}$ In sim formation of

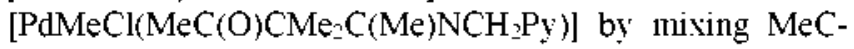
(O) $\left.\mathrm{CMe}_{2} \mathrm{C}(\mathrm{Me}) \mathrm{NCH}_{2} \mathrm{Py}\right)$ with $\mathrm{Pd}(\mathrm{cod}) \mathrm{MeCl}$ confirms the possibility of coordination of keto form of $\beta$-ketoimine. This complex was activated with MMAO to produce polynorbornene but activity ( $48000 \mathrm{~kg} / \mathrm{Pd}$ mol-hr in $\mathrm{CH}_{2} \mathrm{Cl}_{2}$ and 8300 $\mathrm{kg} / \mathrm{Pd}$ mollur in toluene) is much lower than that $(93400 \mathrm{~kg} / \mathrm{Pd}$ molhr in $\mathrm{CH}_{2} \mathrm{Cl}_{2}$ ) with 1+c. Activation with $\mathrm{NaBAr}_{4}^{\prime}$ also produces polynorbornene but activity is not high (630 and 210 $\mathrm{kg} / \mathrm{Pd}$ mollur. respectively). This complex did not show any activity towards olefin polymerization without cocatalysts. Attempts to isolate $\left[\mathrm{PdMeCl}\left(\mathrm{MeC}(\mathrm{O}) \mathrm{CMe}_{2} \mathrm{C}(\mathrm{Me}) \mathrm{NCH}_{2} \mathrm{Py}\right)\right]$ failed due to instability.

However. protonation by $\mathrm{HBF}_{4}$ resulted in the protonation onto pyridine of $\beta$-ketoiminate ligand. (new peak at $9.20 \mathrm{ppm}$ in the $\mathrm{H}$ NMR spectrum) Therefore counter anion appears to be important for the activation of catalyst precursors even though the reason is not clear yet. The catalytic activity of $\mathbf{1 4 c}$ in $\mathrm{CH}_{2} \mathrm{Cl}_{2}$ on activation with $\mathrm{HBF}_{4}(667 \mathrm{~kg} / \mathrm{Pd} \mathrm{mol} \cdot \mathrm{hr})$ is found to be lower than that with $\mathrm{HBAr}^{\circ}(1030 \mathrm{~kg} / \mathrm{Pd}$ nol-hr $)$. In toluene, the activities decrease further to 250 and $+20 \mathrm{~kg} / \mathrm{Pd}$ nol-hr, respectively.

\section{Conclusion}

Three classes of the new palladiun complexes with modified $\beta$-ketoiminate ligands containing pendant bases. [Pd(methallyl)$(\beta$-ketoiminate)]. [Pd(Me)(PPh $)(\beta$-ketoiminate $)]$ and $[\mathrm{Pd}(\mathrm{Me})-$ ( $\beta$-ketoininate)], have been successfully prepared. The structures of three complexes representing the each class were crystallographically deternined which showed that coordination geometries are distorted square planar and internal bases on the $\beta$-ketoiminate ligands fail to coordinate to the metal in the first two classes while bases do coordinate in the class (III)

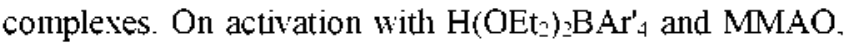
these complexes showed high activities towards norbonene and ethylene polymerization. MMAO is more efficient for the activation for polymerization. Generally. the polymerization activity increases $w$ ith the following order: $[\operatorname{Pd}($ methallyl $)(\beta-$ ketoiminate $)]<\left[\mathrm{Pd}(\mathrm{Me})\left(\mathrm{PPh}_{3}\right)(\beta\right.$-ketoiminate $\left.)\right]<[\mathrm{Pd}(\mathrm{Me})(\beta-$ ketoininate)]. NMR experiments revealed that $\beta$-ketiminates in all classes react with $\left.\left[\mathrm{H}_{(\mathrm{OEt}}\right)_{2}\right]\left[\mathrm{BAr}_{4}\right]$ preferentially to produce active cationic species. In the class (I) and (II) 
complexes, the dissociation of $\beta$-ketoiminates was observed but these ligands were found to be coordinated in the class (III) complexes. Higher polymerization activity was observed in the polar solvent.

Acknowledgments. Authors are grateful for the financial support from Korea Science and Engineering Foundation (KOSEF) (R01-2002-000-00146-0) and I. M. Lee show's his gratitude to both Inha and Hiroshima universities for allowing the sabbatical leave (2004. $9-2005$. 2). Lee also appreciates the invitational fellowship supported by Korea Science and Engineering Funds (KOSEF) and Japan Society for the Promotion of Science (JSPS) (2004).

\section{References}

1. Ittel, S. D.: Jolunson, T. K.; Brookhart, M. Chen Rev. 2000, 100, 1169.

2. Mecking, S. Angew: Chem. Int Ed. 2001, 40, 534.

3. Brunkan, N. M.; Gagne, M. R. Organometallics 2002, 21, 1576 and references therein.

4. Keim, W. Am . N. Acad. Sci. 1983, $+15,191$ and references therein.

5. (a) Johu1son, L. K.; Killian, C. M.; Brookhart, M. J. Am. Chem. Soc. $1995,117,6414$. (b) Rix, F. C.: Brookhart, M.: White, P. S. J.Am. Chem. Soc. 1996, 119, 4746. (c) Mecking, S.; Jolunson, L. K.: Wang, L.: Brookhart, M. J. Am. Chem. Soc. 1998, 120, 888. (d) Svejda, S. A.; Tohnson, L. K. Brookhart, M. J. Ant. Chent. Soc. 1999, 121, 10634. (e) Tempel, D. J.: Tolunson, L. K.; Huff, R. L.: White, P. S.; Brookhart, M. J . im Chem. Soc. 2000, 122, 6686. (f) Schultz, L. H.: Tempel, D. T.: Brookhart, M. J. Ant. Chem. Soc. 2001, 123, 11539 . (g) Schultz, L. H.: Brookhart, M. Organometallics 2001, 20, 3975.

6. (a) Younkin, T. R.; Connor, E. F.: Henderson, I. I.; Friedrich, S.; Grubbs, R. H.; Bansleben, D. A. Science 2000, 287, 460. (b) Wang, C.; Friedrich, S: Younkin, T. R.: Li, R. T: Grubbs, R. H. Basleben. D. A.: Day, M. W. Organometallics 1998, 17, 3149.

7. (a) Britovek, G. J. P.: Gibson, V. C.: Wass, D. F. Angew (Chem. Int. Ed. 1999, 38, 428. (b) Imanishi, Y: Naga, N. Prog. Polyn. Sci. 2001, 26, 1147

8. (a) Male, N. A. H.; Thomton-Pett, M.: Bochmann, M. J. Chem. Soc. Dalton Trans. 1997, 2487. (b) Kooistra. T. M.: Hekking, K. F. W:; Knijnenburg, Q: de Bruin, B.; Budzelaar, P. H. M.: de Gelder, R.; Smits, J. M. M.; Gal, A. W. Etr. J. Inorg. Chent. 2003, 648. (c) Borkar, S.; Saxena, P. K. Polm Bull. 2000, th, 167. (d) Bistovsek, G. J. P.: Bangh, S. P. D.: Hoaran, O; Gibson, V. C.: Wass, D. F.: White, A. T. P.: Williams, D. T. horg. Chim. Acto $2003,345,279$

9. Hennis, A. D.; Polley, J. D.; Long, G. S.; Sen, A.; Yandulov, D.; Lipian, I.: Benedikt, G. M: Rhodes, L. F. Organometallics 2001, 20,2812 and references therein

10. Yang, H.: Li, Z: Sun, W. H. J. Mol Catal .1: Chemical 2003, 206,23 and references therein.

11. (a) Taniak, C. Lassahn. P. G. Macrontol. Rapid Commnth. 2001, 22, 479. (b) Janiak, C; Lassahn, P. G. J. Mol. Catal A. Chemical 2001, 166,193 .

12. (a) Guan, Z.; Cotts, P. M.; McCord, E. F; McLain, S. J. Science 1999, 283, 2059. (b) Held, A.; Ballers, F. M.: Mecking, S. Chent. Commin. 2000,301

13. Lassalun, P. G.: Taniak, C., Oh, J. S. Macromol Rapid Commum. $2002,23,16$ and references therein.

14. (a) Safir, L.; Novak, B. M. Afacronolecules 1995, 28, 5396. (b) Andt, M.: Gosmann, M. Polvm. Bull. 1998, 11,433 . (c) Zhu, Y. Z.: Liu, I. Y:; Li, Y. S.: Tong, Y. I. J. Orgnomet. Chem. 2004, 689,
1295. (d) Zhao, C. T:; Ribeiro, M. R.; Portela, M. F. J. Mol. Catal . A. Chemical 2002, 185, 81. (e) He, X: Yao, Y.: Luo, X:; Zhang, J.: Liu, Y.: Zhang, L.: Wu, Q. Organometallics 2003, 22, 4952. (f) Wang, L. Y.; Li, Y. F; Zhu, F. M: Wu, Q. Europecon Polvm. J. 2006, 12, 322. (g) Bao, F.; Lu, X.; Kang, B; Wu, Q. Enopean Polum J. 2006, 42, 928 (h) Gui, G.; Bao, F.: Gao, H.: Zhu, F, Wu, Q. Appl. Organomet. Chem 2005, 19,627.

15. (a) Min, Y.; Cho, Y. T.; Kim, D.; Lee, J. H.; Kim, B. M.; Lim, S. K. Lee, I. M: Lee, W. I. Chem. Iapor Depos. 2001, 7, 146. (b) Min, Y.; Cho, Y. J.: Kim, D.: Lee, J. H.; Kim, B. M.: Lim, S. K.: Lee, I. M.: Lee, W. I. J. Phws $I F F$, 2001, 11,675 (c) Hong, S. H.; Lim, S. K.: Lee, I. M.: Min, Y.; Kim, D; Lee, W. I. Thin Solid Films 2002, 409,82 . (d) Lim, S. K.; Lee, J. C.; Solu1, D. S.; Lee, W. I. Lee, I. M. Chem. Hater, 2002, 14, 1548 . (e) Lim, S. K. Choi, B. H.: Min, Y.: Kim, D.; Yoon, I.: Lee, S. S.: Lee, I. M. J. Oiganomet. Chem. 2004, 689, 224

16. (a) Bastianini, A.; Battiston, G. A.; Benetollo, F.; Gerbasi, R:; Porchia, M. Polvitedron 1997, 16, 1105. (b) Doherty, S.; Errington, R. J.: Housley, N.: Ridland, I.; Clegg. W.; Elsegood, M. R. I. Oiganometalics 1999, 18, 1018 and references therein. (c) Lee. D. H.; Park, S.-E.; Cho, K.; Kim, Y.; Athar, T.; Lee, I. M. Tetwhedron Lett. $\mathbf{2 0 0 7}, 8281$

17. Neumayer, D. A.: Belot, J. A.; Feezel, R. L.; Reedy, C.; Stern, C. L.; Marks, T. J. Hoig. Chem 1998, $37,5625$.

18. Zhang, Y. P.: Yuan, Z.: Puddephatt, R. J. Chem. Mater. 1998, 10 , 2293.

19. Ladipo, F. T.; Anderson, G. K. Orgonometallics 1994, 13, 303.

20. Rulke, R. E.: Ensting. I. M.; Speck, A. L.: Elsevier, C. J.: van Leenwen, P. W. N. M.: Vrieze, K. Inoig. Chem 1993, 32, 5769.

21. Brookhart, M:- Grant, B.; Volpe, Jr., A. F. Organometallics 1992, 11,3920 .

22. Lee, D. H.: Cho, M. H.: Kwon, I. H.: Jun, I. C.: Lee, I. M.: Jin, M. I. Catal Commun 2008, 9(6), 1517.

23. CCDC-608594(1a) CCDC-608595(tb), and 608596 (12c) contain the supplementary crystallographic data for this paper. These data can be obtained free of charge from the Cambridge Crystallographic Data Centre via www.ccdc.cam.ac.utidata_request: cif. The crystal structure of $t$ b was reported elsewhere. (see supporting information; Lee, D. W.; Jung, J. Y.; Lee, I. M.; Jin, M. T. Eun.J. Org. Chem. 2008, 356.

24. Goddard, R.; Krilger, C.: Mynott, R.; Neumann, M.; Wilke, G. J. Oiganomet. Chem. 1993, 454, C20

25. Lipian, T.; Mimna, R. A.; Fondran, T. C.; Yandulov, D.; Shick, R. A.: Goodall, B. L.; Rhodes, L. F.; Huffman, J. C. Hacrontolecules 2002, 35(24), 8969 .

26. Faller, J. W:; Blankenship, C.: Whitmore, B.: Sena, S. Inoig. Chent $1985,24,4483$

27. Malinoski, J. M: Brookhart, M. Orgonometallics $2003,22,5324$.

28. Daugulis, O.; Brookhart, M. White, P. S. Organontetallics 2002. 21,5935 .

29. Dallgulis, O.: Brookhart, M. Organometallics 2002, 21, 5926.

30. Chen, H. P.; Liu, Y. H.; Peng, S. M.; Liu, S. T. Orgonontetallics 2003, 22, 4893 .

31. Li, K.; Darkwa, T.: Guzei, I.; Mapolie, S. F. J. Organomet. Chem. 2002. 660 . 108 .

32. Müller, G;: Klinga, M.; Osswald, P: Leskela, M:; Rieger, B. Z. Katurforsch., B: Chent. Sci. 2002, 57,803

33. Chen, Y. C.; Chen, C. L.: Chen, T. T.: Liu, S. T. Onganometallics 2001, 20, 1285

34. Reddy, K. R.: Surekha, K.; Lee, G. H.: Peng, S. M.; Chen, I. T.; Liu, S. T. Ongnaontetallics 2000, 19,2637.

35. Liu, W; Brookhart, M. Orgonometallics 2004, 23, 6099

36. (a) van Leenwen, P. W. N. M. Rec. Trav Chim. 1968, 87, 396. (b) Nakamura, Y,; Kawaguchi, S. Chem. Commum 1968, 716. (c) Mehrotra, R. C: Bohra, R: Gaur, D. P. Metal B-Diketonates and Allied Deriwatives; Academic Press: London, England, 1978: p 13 